\title{
Control of Rayleigh-Taylor instability by vertical vibration in large aspect ratio containers
}

\author{
Victoria Lapuerla, Francisco J. Mancebo, and José M. Vega \\ ETSI Aeronáticos, Universidad Politecnica de Madrid, Plaza Cardenal Cisneros, 3, 28040 Madrid, Spain
}

\begin{abstract}
We consider a horizontal heavy fluid layer supported by a light, immiscible one in a wide (as compared to depth) container, which is vertically vibrated intending to counterbalance the Rayleigh-Taylor instability of the flat, rigid-body vibrating state. In the simplest case when the density and viscosity of the lighter fluid are small compared to their counterparts in the heavier fluid, we apply a long wave, weakly nonlinear analysis that yields a generalized Cahn-Hilliard equation for the evolution of the fluid interlace. This ecpuation shows that the slabilizing eflect of vibration is like that of surface tension, and is used to analyze the linear stability of the flat slate, the local bilurcation at the instability threshold and some global existence and stability properties concerning the steady states without dry spots. The analysis is extended to two cases of practical interest. Namely, (a) the viscosity of one of the fluids is much smaller than that of the other one, and (b) the densities and viscosities of both fluids are quite close to each other.
\end{abstract}

I)(OI: 10.1103/PhysRevli.64.016318

PACS number(s): 47.20.Ma, 47.20.Ky

\section{INTRODUCTION AND FORMULATION}

This paper deals with the Rayleigh-Taylor (RT) instability $[1,2]$ (sec also $[3,4]$ and references therein), which appears when a light fluid is accelerated toward a denser one. Thus this instability plays a role in accelerated fronts, which are of interest in, e.g., combustion [5], plasma physics [6], and astrophysics [7]. The analysis of RT instabilities in technological applications such as inertial confinement fusion [6] encounters considerable difficulties because this instability often exhibits a transient nature and/or comes into play in nonstatic conditions, involving convection, heat flow, mass ablation, and inhomogeneities, which affect the instability growth rate. In order to avoid these and deal with a clean formulation, amenable to analytical treatment, we consider the simplest configuration cxhibiting this instability, namely, that in which a horizontal heavy lluid layer is supported by a lighter fluid, the destabilizing acceleration being provided by gravity. In this configuration, the instability can be counterbalanced by an imposed vertical vibration of the container. as already shown experimentally [8,9]; see also [9-11] for a first theoretical explanation. The main object of this paper is to provide a weakly nonlinear theory of this stabilizing effect in the limiting case when both the aspect ratio of the container and the vibrating frequency are appropriately large. Let us mention here that to our knowledge no consistently simplified evolution equations like the ones derived below, accounting for both nonlinearity and viscous effects, are found in the literature for the evolution of the RT instability in the presence of vibration; and similar cvolution equations in nonvibrating systems are of limited scope [4].

Although we shall deal with a more general situation in Sec. IV, in order to illustrate both the analysis and the results, we first consider in Secs. II and III the limiting case in which the lighter fluid can be ignored, which is justified when its density and viscosity are small compared to their counterparts, $\rho$ and $\nu$, in the heavier lluid. Thus we consider a wide cylindrical container of horizontal size $\ell$ and depth $h \leqslant \ell$, which is vertically vibrated and placed in inverted position (see Fig. 1), with gravity acting downwards. We use the depth $h$ and the viscous time $h^{2} / \nu$ as characteristic length and time for nondimensionalization and a Cartesian coordinate system attached to the container, with the $z-0$ plane on the unperturbed free surface, assumed to be horizontal. The (nondimensional) governing equations are

$$
\boldsymbol{\nabla} \cdot \mathbf{u}+\partial w / \partial z-0
$$

$$
\partial \mathbf{u} / \partial t|(\mathbf{u} \cdot \boldsymbol{\nabla}) \mathbf{u}| w d \mathbf{u} / \partial z=\nabla p|\Delta \mathbf{u}| \partial^{2} \mathbf{u} / \partial z^{2},
$$

$\partial w / \partial t+\mathbf{u} \cdot \nabla w+w \partial w / \partial z--\partial p / \partial z+\Delta w+\partial^{2} w / \partial z^{2}$

if $(x, y) \in \Omega$ and $f(x, y, t)<z<1$, with boundary conditions

$$
\begin{gathered}
\mathbf{u}-\mathbf{0}, w-0 \text { if } z-1 \text { and if }(x, y) \in \partial \Omega, \\
w-\partial f / \partial t+\mathbf{u} \cdot \nabla f
\end{gathered}
$$

$$
\begin{gathered}
\partial \mathbf{u} / \partial z \mid \nabla w=O\left(\left.|\nabla \mathbf{u}||\nabla f||(|\partial \mathbf{u} / \partial z||| \nabla w \mid)| \nabla f\right|^{2}\right) \\
\text { if } z-f \\
p-a \omega^{2} f \cos (\omega t)-B C{ }^{2} f-C{ }^{2} \nabla \cdot\left[\nabla f /\left(1+|\nabla f|^{2}\right)^{1 / 2}\right] \\
-2 \partial w / \partial z+O(|\nabla \mathbf{u}|+(|\partial \mathbf{u} / \partial z|+|\nabla w|)|\nabla f|) \text { if } z-f,
\end{gathered}
$$

$$
\boldsymbol{\nabla} f \cdot \mathbf{n}=D \partial f / \partial t \text { or } f=0 \text { if }(x, y) \in \partial \Omega
$$$$
\int_{\Omega} f(x, y, t) d x d y-0
$$

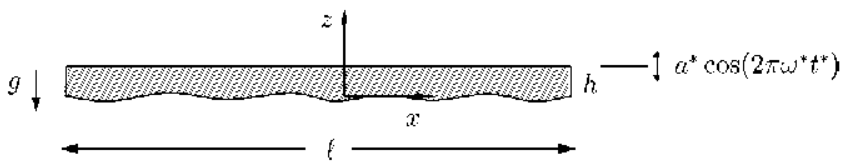

FIG. 1. Sketch of the side view of the container. 


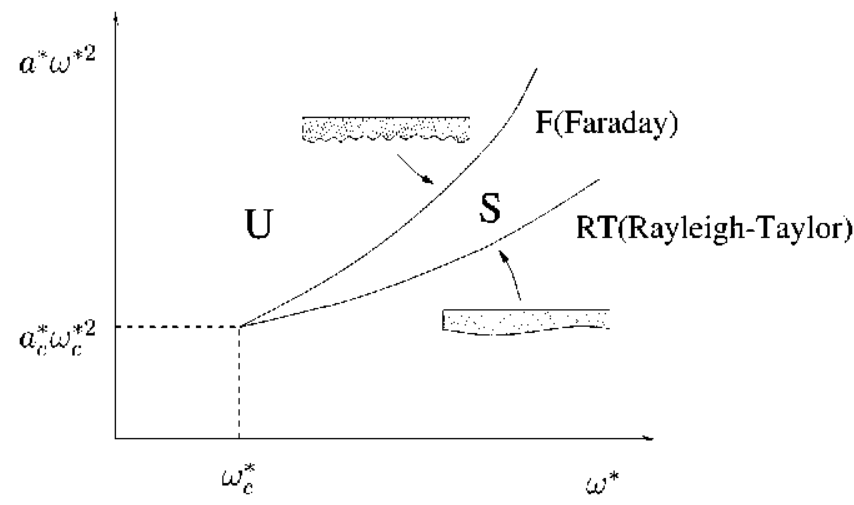

FIG. 2. A typical linear stability diagram of the static steady state (1.11). The regions where this flat state is stable (S) and unstable $(U)$ are indicated.

where $\mathbf{u}$ and $w$ are the horizontal and vertical velocity, $p$ $=$ pressure $\mathrm{I}\left[a \omega^{2} \cos (\omega t) \mid B / C^{2}\right] z$ is a modified pressure and $f$ is the vertical free-surface deflection, assumed along the paper to be such that

$$
|\nabla f| \ll|f|
$$

$\boldsymbol{\nabla}, \boldsymbol{\nabla}$, and $\Delta$ are the horizontal gradient, divergence, and Laplacian operators, $\Omega$ is the transversal cross section of the container, $\partial \Omega$ is its boundary, and $\mathbf{n}$ is the (horizontal) outward unit normal to $\partial \Omega$. The domain $\Omega$ is large and homothetic to a fixed two-dimensional (2D) domain; the (dimensionless) characteristic size of $\Omega$,

$$
L=\mathscr{H} / h \gg 1 \text {, }
$$

is the aspect ratio of the container and

$$
B=\rho g h^{2} / \sigma \text { and } C=\nu \sqrt{\rho /(\sigma h)}
$$

are the Bond number and the capillary number respectively; here $g$ is the gravitational acceleration and $\sigma$ is the surface tension cocflicient. In the first boundary condition (1.7) cither we allow a contact line motion or not. In the former case we assume that the static contact angle is 9()$^{\circ}$ and cmploy the usual phenomenological law (sce, c.g., $[12,13]$ ) to account for contact line dynamics; the phenomenological constant $l$ ) is positive and thus the motion of the contact line is dissipative. And when the contact line is fixed (at the upper edge of the lateral wall) we assume that the height of the lateral wall is $h$ and the liquid volume equals $h$ times the area of $\Omega$. Note that the rigid-body oscillating, flat state

$$
\mathbf{u}=\mathbf{0}, \quad w=p=0, f=0
$$

is a (steady) solution of Eqs. (1.1)-(1.7) in both cases. The linear stability diagram of this solution is always like that sketched in Fig. 2, where

$$
a_{R i} \omega^{1 / 2} \rightarrow K^{*}>0 \quad \text { and } \quad a_{R T} \omega \rightarrow K_{*}>0 \quad \text { as } \omega \rightarrow \infty \text {. }
$$

The upper and lower marginal instability curves correspond to the Faraday instability and the RT instability, respec- tively, which are considered in Sec. II A and Sec. II B. The analysis in this paper applies in the stable region of Fig. 2, outside a neighborhood of the upper instability boundary; thus the eigenmodes associated with the Faraday instability are exponentially stable and can be ignored.

Figure 2 is qualitatively similar to the one obtained experimentally by Wolf [9] and illustrates that stabilization is always feasible provided that $\omega$ is sufficiently large. Of course, the forcing frequency is bounded in practice to no higher than ultrasound frequencies (say, $\leqslant 20 \mathrm{kHz}$ ); a second limil results from the mechanical difficulties in imposing 100 large an accelcration (note that the nondimensional acceleration $a \omega^{2}$ grows with $\omega$ along the lower bound of the stable region in Fig. 2).

Thus we shall be mainly concerned below with the limit $\omega \rightarrow \infty$. But for simplicity we shall begin in Sec. II A with the linear stability analysis of the basic steady state (1.11) in the viscous limit

$$
B L^{2} \cdots C \cdots D / L^{4} \sim a \cdots \omega \cdots 1
$$

which yields the most general results because in this limit no further approximation is made (in addition to linearization). That analysis will also be valid for large $\omega$ and will help us to identify the distinguished limit

$$
B L^{2} \sim a \omega C \cdots a^{2} \omega^{2} D / L^{3} \cdots 1, \quad \omega \geqslant 1,
$$

which is the limit that provides the most general results for large forcing frequency. This limit will be considered in Sec. III, where the leading nonlinear terms will also be taken into account to obtain an evolution equation for the free surface deflection. Finally, the more general case of a two-fluid layer will be considered in Sec. IV, where for simplicity the final form of the cvolution equation accounting for weakly nonlincar dynamics will be only derived in two limiting cases, namely, that in which one of the fluids is inviscid compared to the other one, and that in which the densities and viscositics of both fluids are almost equal.

\section{LINEAR STABILITY OF THE FLAT STATE}

Let us linearize Eqs. (1.1)-(1.7) around the basic state (1.11) to obtain

$$
\begin{gathered}
\boldsymbol{\nabla} \cdot \mathbf{u}+\partial w / \partial z-0, \partial \mathbf{u} / \partial t--\nabla p+\Delta \mathbf{u}+\partial^{2} \mathbf{u} / \partial z^{2} \\
\quad \partial w / \partial t--\partial p / \partial z+\Delta w+\partial^{2} w / \partial z^{2} \\
\text { if }(x, y) \in \Omega \text { and } 0<z<1 \text {, and } \\
\mathbf{u}-\mathbf{0}, \quad w-0 \text { if } z-1 \text { and if }(x, y) \in \partial \Omega, \\
w-\partial f / \partial t, \partial \mathbf{u} / \partial z+\nabla w-0 \text { if } z-0, \\
p-a \omega^{2} f \cos (\omega t)-B C^{-2} f-C^{-2} \Delta f-2 \partial w / \partial z \text { if } z-0
\end{gathered}
$$

$$
\boldsymbol{\nabla} f \cdot \mathbf{n}--D \partial f / \partial t \text { or } f-0 \text { if }(x, y) \in \partial \Omega,
$$




$$
\int_{\Omega} f(x, y, t) d x d y=0
$$

As anticipated in Sec. I and illustrated in Fig. 2, marginal instability occurs at two possible type of modes, which exhibit short and large wavelengths, of the order of the depth and the width of the container, respectively.

\section{A. Short-wave instability: Faraday waves}

This instability, named after Faraday [14], has been thoroughly studied [15-17]. In the limits (1.13) and (1.14) the most unstable modes exhibit a wavelength that is at the most of the order unity. Since the container cross section is large, end-wall cffects are usually ignored, and the stability analysis of Eqs. (2.1)-(2.5) is made by only considering the normal modes, which are of the form

$$
(\mathbf{u}, w, p, f)-(\mathbf{U}, W, P, F) \exp \left[i\left(k_{1} x+k_{2} v\right)\right]+\text { c.c., }
$$

where $\mathbf{U}, W$, and $P$ depend only on $z$ and $t$, and $F$ depends only on $t$ and c.c. denotes the complex conjugate. Substitution of these expressions into Eqs. (2.1)-(2.5) and elimination of $\mathbf{U}$ yield

$$
\begin{gathered}
P_{z z}-k^{2} P, W_{t}--P_{z}+W_{z z}-k^{2} W, \\
W-W_{z}-0, \text { at } z--1, \\
W \quad F_{t}=W_{z z} \mid k^{2} W=0, \text { at } z=0, \\
P-a \omega^{2} F^{\prime} \cos (\omega t)-\left(B-k^{2}\right) H / C^{2}-2 W_{z} \text {, at } z-0,
\end{gathered}
$$

where $k-\sqrt{k_{1}^{2}+k_{2}^{2}}$ is the wave number of the mode. The calculation of the instability threshold forcing amplitude $a_{F}$ requires to determine those Floquet exponents of Eqs. (2.7)(2.10) whose real part vanishes; in fact, these exponents are numerically found to be either 0 or $i \pi$, which correspond to real Floquet multipliers 1 or -1 , respectively. For fixed values of the remaining parameters, this determines a curve $a-k$ whose absolute minimum yields $a_{k}$. The numerical calculation of the Floquet exponents is fairly cheap, even for extreme values of the parameters, by using the method described in Ref. [18]. The problem still depends on $\omega, B$, and $C$, which makes its analysis fairly tedious. For the sake of brevity we only give results here for sufficiently large forcing frequency, namely,

$$
1+\left(B / C^{2}\right)^{2 / 3} \ll \omega
$$

which is the more convenient one for the main object of this paper. In this limit, gravity can be neglected and the marginally unstable modes exhibit a short wavelength $k^{1} \cdots \omega^{1 / 2}$ $\ll 1$. As a consequence, Eqs. (2.7)-(2.10) can be further rescaled to obtain a new problem that only depends on a rescaled wave number $\omega^{-1 / 2} k$ and on the parameters $a \sqrt{\omega}$ and $C^{4} \omega$. Using the latter two parameters, we numerically obtain the instability threshold curve plotted in Fig. 3, which pro-

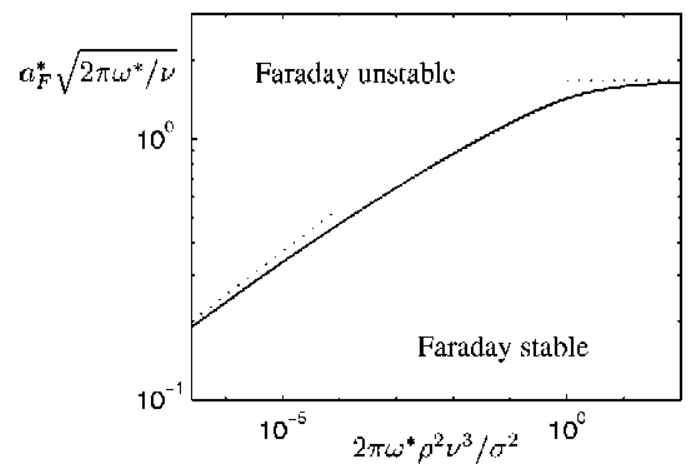

]']G. 3. A plot of the rescaled laraday instability threshold amplitude $a_{F} \omega^{1 / 2} \equiv\left(2 \pi \omega^{*} / \nu\right)^{1 / 2} a_{F}^{*}$ in terms of $C^{4} \omega \equiv 2 \pi \omega^{*} \rho^{2} \nu^{3 /} / \sigma^{2}$ in the limit (2.11); the asymptotes as $C^{4} \omega \rightarrow 0$ and $C^{4} \omega \rightarrow \infty$ are plotted with dotted lines.

vides the upper instability limit in the sketch in Fig. 2. Note that this curve satisfies the first condition (1.12), with

$$
K^{*} \simeq 1.67
$$

Let us point out here that the additional requirement $\left(B / C^{2}\right)^{2 / 3} \ll \omega$ in Eq. (2.11) is seen to be automatically satisfied in the stable region sketched in Fig. 2 when the upper boundary is as calculated here and the lower one is as given by $\mathrm{Eq} .(2.40)$ below. Thus this additional condition is unnecessary when seeking stability in the limit $\omega \gg 1$.

\section{B. Long-wave instability: Rayleigh-Taylor}

As is well known [3], in the absence of vibration (if $a$ -0 ) the linear problem (2.1)-(2.5) exhibits exponential instability (i.e., the RT instability) whenever

$$
\widetilde{B} \equiv B L^{2}>\lambda_{0},
$$

where $\lambda_{0}$ is the lowest eigenvalue of,

$$
\begin{gathered}
\tilde{\Delta} F^{\prime}+\lambda F^{\prime}-\text { const in } \widetilde{\Omega}, \partial F / \partial \tilde{n}-0 \text { or } F^{\prime}-0 \text { on } \partial \tilde{\Omega} \\
\int_{\tilde{\Omega}} F d \tilde{x} d \tilde{y}=0,
\end{gathered}
$$

with

$$
\widetilde{\Delta}-\partial^{2} / \partial \tilde{x}^{2}+\partial^{2} / \partial \tilde{y}^{2}, \tilde{x}-x / h, \tilde{y}-y / L ;
$$

$\widetilde{\Omega}$ is the result of rescaling $\Omega$ with $L$ and $\partial / \partial \tilde{n}$ is the resulting derivative along the outward unit normal. For the simplest cross sections, namely, the circle of diameter $1, \widetilde{\Omega}_{1}$ and the rectangle of sides $d \geqslant 1$ and $1, \widetilde{\Omega}_{2}$, the lowest eigenvalue $\lambda_{0}$, and an associated eigenfunction $F_{0}$ are given by

$$
\begin{gathered}
\lambda_{0}=4 \gamma_{1}^{2}, \quad F_{0}=J_{1}\left(2 \gamma_{1} r\right) \cos \left(\theta \quad \theta_{0}\right) \text { in } \widetilde{\Omega}_{1}, \\
\text { and } \lambda_{00}=\pi^{2} / d^{2}, \quad F_{0}=\cos (\pi \tilde{x} / d) \text { in } \widetilde{\Omega}_{2}
\end{gathered}
$$

if the first boundary condition in Eq. (2.14) applies, where $\gamma_{1} \simeq 2.40$ is the first positive root of the derivative $J_{1}^{\prime}$ of the 
first Bessel function $J_{1}, r$ and $\theta$ are polar coordinates, and $\theta_{0}$ is an arbitrary constant resulting from invariance under rotation; and

$$
\begin{gathered}
\lambda_{0}=4 \gamma_{2}^{2}, \quad F_{0}=J_{1}\left(2 \gamma_{2} r\right) \cos \left(\theta \quad \theta_{0}\right) \text { in } \widetilde{\Omega}_{1}, \\
\lambda_{0}=\pi^{2} \mid 4 \pi^{2} / d^{2}, \quad F_{0}=\sin (2 \pi \tilde{x} / d) \sin (\pi \tilde{y}) \text { in } \widetilde{\Omega}_{2},
\end{gathered}
$$

if the second boundary condition in Eq. (2.14) applies, where $\gamma_{2}=3.83$ is the first positive root of $J_{1}$. Note that all these eigenfunctions are antisymmetric on a straight line $(\tilde{x}=d / 2$ in $\widetilde{\Omega}_{1}$ or $\theta-\theta_{0} \mid \pi / 2$ in $\widetilde{\Omega}_{2}$ ). There are also symmetric eigenfunctions, but they are associated with larger eigenvalues.

Thus the instability sets in for $O\left(L^{2}\right)$ values of the Bond number $B$. Viscosity does not affect the instability threshold and the only stabilizing effect results from the surface tension. In fact, the stabilizing effect of vibration in this limit is to "create a surface-tension-like" mechanism as we show now. To this end, we consider the viscous limit (1.13) and use a two-time-scales method as follows. In the distinguished limit (1.13) we rescale the Bond number and the horizontal space variables as in Eqs. (2.13) and (2.15), introduce the slow lime variable

$$
\tilde{t}=t / L^{4}
$$

and seek solutions of Eqs. (2.1)-(2.5) of the form

$$
\begin{aligned}
& \mathbf{u}-L{ }^{1} \mathbf{u}_{o}(\tilde{x}, \tilde{y}, z, \tilde{t}) e^{i m t}+L{ }^{3} \text { h.o.h.+c.c. } \\
& +l^{-3} \mathbf{u}_{s}(\tilde{x}, \tilde{y}, z, \tilde{l})+\cdots, \\
& w=L^{-2} w_{o}(\tilde{x}, \tilde{y}, z, \tilde{t}) e^{i \omega t} \mid L^{-4} \text { h.o.h. । c.c. } \\
& +L^{-4} w_{s}(\tilde{x}, \tilde{y}, z, \tilde{t})+\cdots, \\
& p-p_{o}(\tilde{x}, \tilde{y}, z, \tilde{t}) e^{i \omega t}+L^{-2} \text { h.o.h. }+ \text { c.c. } \\
& \left|L^{-2} p_{s}(\tilde{x}, \tilde{y}, z, \tilde{t})\right| \cdots, \\
& f=L \quad{ }^{2} f_{o}(\tilde{x}, \tilde{y}, \tilde{t}) e^{i \omega t} \mid L{ }^{4} \text { h.o.h. | c.c. }\left|f_{s}(\tilde{x}, \tilde{y}, \tilde{t})\right| \cdots \text {, }
\end{aligned}
$$

where c.c. denotes the complex conjugate and h.o.h. stands for higher order harmonics, depending on the fast time variable $t$ as $e^{i m \omega t}$, with $m \neq 0, \pm 1$. The scalings (2.19) are obtained by an orders-of-magnitude analysis in Eqs. (2.1)(2.5), anticipating that in the absence of vibration the dispersion relation of the long-wave modes of Eqs. (2.7)(2.10) associated with the RT instability is $\mu$ (三 growth rate) $-\left(B-k^{2}\right) k^{2} /\left(3 C^{2}\right)+O\left(k^{6}\right)$ as $k \rightarrow 0$. Substituting Eq. (2.15) and Eqs. (2.18) and (2.19) into Eqs. (2.1)-(2.4) and the last equation in Eq. (2.5) yields

$$
\begin{gathered}
\widetilde{\boldsymbol{\nabla}} \cdot \mathbf{u}_{o}+\partial w_{o} / \partial z-0, \quad i \omega \mathbf{u}_{o}--\widetilde{\nabla} p_{o}+\partial^{2} \mathbf{u}_{o} / \partial z^{2} \\
\partial p_{o} / \partial z-0
\end{gathered}
$$

$$
\widetilde{\mathbf{\nabla}} \cdot \mathbf{u}_{s}+\partial w_{s} / \partial z-0, \quad \widetilde{\nabla} p_{s}-\partial^{2} \mathbf{u}_{s} / \partial z^{2}, \partial p_{s} / \partial z-0,
$$

if $(x, y) \in \Omega$ and $0<z<1$, and

$$
\begin{gathered}
\mathbf{u}_{o}-\mathbf{u}_{s}-\mathbf{0}, \quad w_{o}-w_{s}-0 \text { if } z-1, \\
w_{o}=i \omega f_{o}, \quad w_{s}=\partial f_{s} / \partial \tilde{t}, \quad \partial \mathbf{u}_{o} / \partial z=\partial \mathbf{u}_{s} / \partial z=\mathbf{0} \text { if } z=0, \\
p_{o}=a \omega^{2} f_{s} / 2, \\
p_{s}-a \omega^{2}\left(f_{o}+\overline{f_{o}}\right) / 2+\widetilde{B} C^{-2} f_{s}+C^{-2} \widetilde{\Delta} f_{s} \text { if } z-0 \\
\int_{\tilde{\Omega}} f_{s} d \tilde{x} d \tilde{y}-0,
\end{gathered}
$$

where $\widetilde{\Omega}, \widetilde{\nabla}$, and $\widetilde{\Delta}$ are as defined above and the overbar denotes the complex conjugate. Equations (2.20)-(2.24) do not apply in a boundary layer of $O(1)$ thickness near the lateral walls. The analysis of this boundary layer (see Appen$\operatorname{dix}$ A) provides the following boundary conditions for the solution in the bulk:

$$
\partial f_{s} / \partial \tilde{n}--\tilde{D} \partial f_{s} / \partial \tilde{t} \text { or } f_{s}-0, \int_{0}^{1} \mathbf{u}_{s} \cdot \tilde{\mathbf{n}} d z-0 \text { on } \partial \widetilde{\Omega}
$$

where $\tilde{\mathbf{n}}$ is the unit outward normal to $\partial \widetilde{\Omega}$ as above,

$$
\widetilde{D}-2 D /\left[\left\{2+a^{2} \omega^{2} C^{2} \phi(\omega)\right\} L^{3}\right],
$$

and the function $\phi$ is defined as

$$
\phi(\omega)-1-\operatorname{Rc}\left((\sqrt{i \omega})^{-1} \tanh \sqrt{i \omega}\right)
$$

with Re standing for the real part. Note that

$$
\phi(\omega)>0 \text { for all } \omega>0 \text {, and } \phi(\omega) \rightarrow 1 \text { as } \omega \rightarrow \infty .
$$

Integration of Eqs. (2.20)-(2.24) yields

$$
\begin{gathered}
p_{o}-a \omega^{2} \int_{s} / 2, \\
\mathbf{u}_{o}=i(a \omega / 2)\left[1 \quad(\cosh \sqrt{i \omega})^{-1} \cosh \sqrt{i \omega} z\right] \widetilde{\nabla} f_{s}, \\
w_{o}-i(a \omega / 2)\left[1-z-(\sqrt{i \omega} \cosh \sqrt{i \omega})^{-1}\right. \\
\times(\sinh \sqrt{i \omega} \sinh \sqrt{i \omega z})] \widetilde{\Delta} f_{s}, \\
f_{o}-(a / 2)[1-(\sqrt{i \omega}) \quad \tanh \sqrt{i \omega}] \widetilde{\Delta} f_{s}, \\
\mathbf{u}_{s}-\left(z^{2}-1\right) \widetilde{\nabla} p_{s} / 2, w_{s}--\left(2-3 z+z^{3}\right) \widetilde{\Delta} p_{s} / 6, \\
\partial f_{s} / \partial \tilde{t}=\widetilde{\Delta}_{s} / 3 \\
p_{s}-\widetilde{B} C{ }^{2} f_{s}+\left[C{ }^{2}+a^{2} \omega^{2} \phi(\omega) / 2\right] \widetilde{\Delta} f_{s},
\end{gathered}
$$




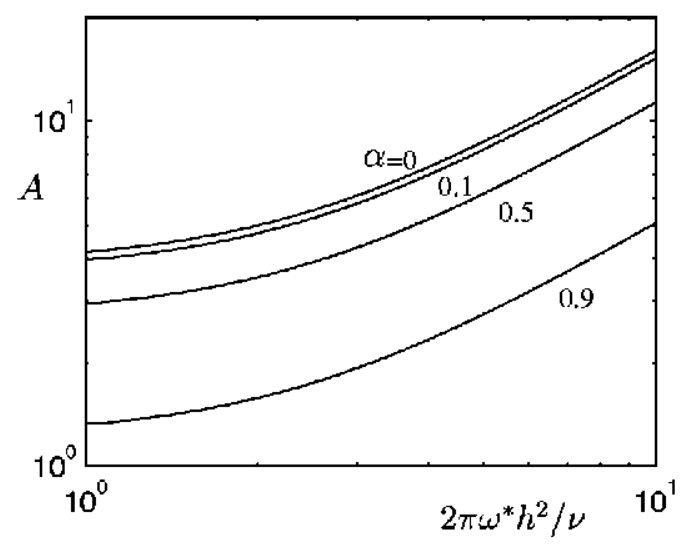

IIG. 4. A plot of the rescaled R'l instability threshold acceleration, $A-\left[\sqrt{\lambda_{0}} C /(\sqrt{B} L)\right] a_{R T} \omega^{2} \equiv\left[\lambda_{0}^{1 / 2} h^{5 / 2} /\left(\ell g^{1 / 2} \nu\right)\right] a_{R T}^{*}\left(2 \pi \omega^{*}\right)^{2}$, in terms of the nondimensional forcing frequency, $\omega=2 \pi \omega^{*} h^{2 /} \nu$, for the indicated values of the parameter $a-\lambda_{0} /\left(B L^{2}\right)$ $\equiv \lambda_{0} \sigma /\left(\rho g t^{\prime 2}\right)$, as given by Eq. (2.38).

where the function $\phi$ is as defined in Eq. (2.28). And a further substitution of Eq. (2.34) into the last equation in Eq. (2.33) leads to

$$
\begin{aligned}
& 3 \partial f_{s} / \partial \tilde{t}\left|\widetilde{B} C^{-2} \widetilde{\Delta} f_{s}\right|\left[C^{-2} \mid a^{2} \omega^{2} \phi(\omega) / 2\right] \widetilde{\Delta}^{2} f_{s} \\
& \quad-0 \text { in } \widetilde{\Omega},
\end{aligned}
$$

where $\widetilde{\Delta}^{2}$ stands for the biharmonic opcrator. In addition, we must use the volume conservation condition (2.25) and the boundary conditions (2.26) that, using the first expression (2.33), arc

$$
\begin{aligned}
& \partial f_{s} / \partial \tilde{n}--\tilde{D} \partial f_{s} / \partial \tilde{t} \text { or } f_{s}-0, \\
& \tilde{B} C^{-2} \partial f_{s} / \partial \tilde{n}+\left[C^{-2}+a^{2} \omega^{2} \phi(\omega) / 2\right] \partial \tilde{\Delta} f_{s} / \partial \tilde{n}-0 \\
& \text { on } \partial \widetilde{\Omega}, \quad \int_{\tilde{\Omega}} f_{s} d \tilde{x} d \tilde{y}-0 .
\end{aligned}
$$

Note that, as anticipated above, the effect of vibration [measured by the term $a^{2} \omega^{2} \phi(\omega)$ ] is equivalent to enhancing the effective surface tension of the free surface, measured by $\mathrm{C}^{-2}$ in nondimensional terms. According to the analysis in Appendix B, this solution is asymptotically stable, if and only if

$$
B L^{2} \equiv \widetilde{B}<\widetilde{B}_{c} \equiv \lambda_{0}\left[1+C^{2} a^{2} \omega^{2} \phi(\omega) / 2\right],
$$

where $\lambda_{0}$ is the lowest eigenvalue of Eq. (2.14). Thus, the instability threshold amplitude $a_{R T}$ is given by

$$
\left[\lambda_{0} C^{2} /\left(B L^{2}\right)\right] a_{R T}^{2} \omega^{2}=2\left[\begin{array}{ll}
1 & \lambda_{0} /\left(B L^{2}\right)
\end{array}\right] / \phi(\omega)
$$

and yields the threshold acceleration plotted vs $\omega$ in Fig. 4 for several values of the parameter $\lambda_{0} /\left(B L^{2}\right)$, which must be such that $0 \leqslant \lambda_{0} /\left(B L^{2}\right)<1$, in order that the system is unstable in the absence of vibration (otherwise, the RT instability does not appear). Note that, according to the nondi- mensionalization in Sec. I, $\lambda_{0} /\left(B L^{2}\right)-\lambda_{0} \sigma /\left(\rho g \ell^{2}\right)$. If the surface tension is not much larger and the density is not much smaller than those of the water, this parameter is small whenever the horizontal size of the container is large compared to the capillary length $(\sqrt{\sigma /(\rho g)} \sim 3 \mathrm{~mm})$.

Some remarks about this result are now in order:

(1) The stabilizing effect of vibration is like that of surface tension, which can be completely substituted by vibration. In the absence of surface tension $C^{2} \sim B \rightarrow \infty$ and the instability threshold is given by the highest curve in Fig. 4 . The origin of this stabilizing effect is clear from the solution (2.30)-(2.34) of Eqs. (2.20)-(2.24). If a (nonconstant) free surface deflection $f_{s}$ is present then the system cannot vibrate as a rigid body and an oscillating flow appears whose associated free surface deflection is proportional to $a \tilde{\Delta} f_{s}$ [see Eq. (2.32)] and in turn produces [through nonlinear interaction with the primary oscillating pressure field $-a \omega^{2} z \cos (\omega t)$, accounted for in the second term in the lefthand side of Eq. (2.4)] a nonoscillating overpressure proportional to $a^{2} \omega^{2} \widetilde{\Delta} f_{s}$; this is the stabilizing term.

(2) The new instability threshold $\tilde{B}_{0}$ given in Eq. $(2.37)$ is higher than that in the absence of vibration, which is $\lambda_{i j}$. For fixed values of the remaining parameters, the plot $a_{R T} \omega^{2}$ vs. $\omega$ in Fig. 4 is as sketched in the lower curve in Fig. 2 and satisfies Eq. (1.12), with $\left.K_{*}=\sqrt{2\left(B L^{2}\right.} \lambda_{0}\right) /\left(\lambda_{0} C^{2}\right)$.

(3) If $a$ and $\omega$ are bounded then, according to Eq. (2.37) stabilization is only possible if $B L^{2}$ is bounded, which means [see Eq. (1.9)] that $B$ must be quite small, and this requires that the depth be extremely small on earth conditions. Condition (2.37) is written in dimensional terms (see Sec. I) as

$$
\ell^{2}<\lambda_{0}\left[\sigma /(\rho g)+\left(2 \pi a^{*} \omega^{*}\right)^{2} h \phi\left(2 \pi \omega^{*} h^{2} / \nu\right) /(2 g)\right]
$$

where $a^{*}$ and $\omega^{*}$ are the dimensional forcing amplitude and frequency. Thus the instability limit depends on viscosity $(\nu)$ through the argument of the function $\phi$. This is not surprising because the stabilization due to vibration is due to oscillations in the bulk that are viscous if $\omega-2 \pi \omega^{*} h^{2} / \nu$ is bounded. In particular, as $\nu$ increases the viscous lime $h^{2} / \nu$ decreases, and the forcing frequency $\omega^{*}$ must also increase to maintain the stabilizing effect of vibration. The situation is much better as $a \omega \rightarrow \infty$, which is easily achieved in the realistic limit $\omega \rightarrow \infty$, when [ $\phi(\omega) \rightarrow 1$, see Eq. (2.29), and] conditions (2.37) and (2.39) become

$$
B L^{2}<\lambda_{0}\left[1 \mid C^{2} \alpha^{2} \omega^{2} \phi(\omega) / 2\right]
$$

and

$$
\mathscr{H}^{2}<\lambda_{0}\left[\sigma /(\rho g)+\left(2 \pi a^{*} \omega^{*}\right)^{2} h / 2 g\right] .
$$

This condition is independent of viscosity [which in this limit $\omega \gg 1$ only comes into play through the Faraday instability, sce remark (4) below]. The reason is that now the oscillatory flow that produces the stabilizing effect is inviscid except in boundary layers, which only produce a higher order effect. If, for illusiration, we consider a circular container of diameter $\ell-5 \mathrm{~cm}$ and depth $h-5 \mathrm{~mm}$ (thus the 
aspect ratio $\mathscr{C} / h-10$ is large) and assume that the contact line is fixed, then $\lambda_{0}=4 \gamma_{2}^{2} \simeq 58.7$ [see Eq. (2.17)] and the condition (2.40) is satisfied provided that

$$
\sigma / \rho \ll 418 \mathrm{~cm}^{3} / \mathrm{s}^{2} \text { and } 2 \pi a^{*} \omega^{*}>40.9 \mathrm{~cm} / \mathrm{s} \text {. }
$$

(4) In addition, we must avoid the Faraday instability by requiring that $a \sqrt{\omega}$ is below the curve in Fig. 3; the validity conditions (2.11) are seen to be satisfied for both mineral oil and water. For a sufficiently large forcing frequency, Faraday waves are avoided, provided that $a \sqrt{\omega<K^{*}}$, which is written in dimensional terms as $2 \pi \omega^{*}\left(a^{*}\right)^{2} \leqslant K^{* 2} \nu$, and this is compatible with Eq. (2.41) only if $2 \pi \omega^{*} \nu \geqslant 600 \mathrm{~cm}^{2} / \mathrm{s}^{2}$. This condition is satisfied for mineral oil $\left(\nu-1 \mathrm{~cm}^{2} / \mathrm{s}\right)$ if, say, $\omega^{*}-10^{2} \mathrm{H} \%$ and $a^{*} \simeq 0.6 \mathrm{~mm}$, and for clean water $(\nu$ $-0.01 \mathrm{~cm}^{2} / \mathrm{s}$ ), if $\omega^{*}-10^{4} \mathrm{H} \%$ and $a^{*}-6 \mu \mathrm{m}$. Of course the situation is much better both in microgravity conditions and when the liquid layer is supported by another layer of liquid of nonzero density. The latter case will be considered in Sec. IV.

(5) The analysis above has the obvious limitations of any linear theory. Nonlinear stability will be analyzed below.

\section{WEAKLY NONLINEAR THEORY FOR LARGE VIBRATING FREQUENCY} that

According to remark (3) at the end of Sec. II we assume

$$
\omega \gg 1 \text { and } a \omega \geqslant 1 \text {. }
$$

\section{A. Asymptotic derivation of an evolution equation for the free surface}

According to Eqs. (2.29)-(2.34), in order that all the terms in Eq. (2.35) be of the same order in the limit (3.1), the following rescaled parameters and slow time variable

$$
\begin{gathered}
\hat{B}=B L^{2}, \quad \hat{C}=a \omega C \sim 1, \\
\hat{D}=2 a^{2} \omega^{2} D /\left[\left(2 \mid a^{2} \omega^{2} C^{2}\right) L^{3}\right] \sim 1, \quad \hat{t}=a^{2} \omega^{2} t / L^{4},
\end{gathered}
$$

must be of order unity. Thus we replace the expansions (2.19) by

$$
\begin{aligned}
\mathbf{u}= & a \omega L^{-1} \mathbf{u}_{o}(\tilde{x}, \tilde{y}, z, \hat{t}) e^{i \omega t} \mid a^{2} \omega L^{-3} \text { h.o.h. । c.c. } \\
& +a^{2} \omega^{2} L^{-3} \mathbf{u}_{s}(\tilde{x}, \tilde{y}, z, \hat{t})+\cdots, \\
w- & a \omega L^{-2} w_{o}(\tilde{x}, \tilde{y}, z, \hat{t}) e^{i \omega t}+a^{2} \omega L^{-4} \text { h.o.h. }+ \text { c.c. } \\
& +a^{2} \omega^{2} L^{-4} w_{s}(\tilde{x}, \tilde{y}, z, \hat{t})+\cdots, \\
p= & a \omega^{2} p_{o}(\tilde{x}, \tilde{y}, z, \tilde{t}) e^{i \omega t} \mid a^{2} \omega^{2} L^{-2} \text { h.o.h. | c.c. } \\
& +a^{2} \omega^{2} L{ }^{2} p_{s}(\tilde{x}, \tilde{y}, z, \hat{t})+\cdots, \\
f-a L{ }^{2} & f_{o}(\tilde{x}, \tilde{y}, \hat{t}) e^{i \omega t}+a^{2} L{ }^{4} \text { h.o.h. }+ \text { c.c. }+f_{s}(\tilde{x}, \tilde{y}, \hat{t})+\cdots,
\end{aligned}
$$

which are now substituted into the original nonlinear problem $(1.1)-(1.6)$, to obtain

$$
\begin{gathered}
\tilde{\boldsymbol{\nabla}} \cdot \mathbf{u}_{o} \mid \partial w_{o} / \partial z=0, \quad i \mathbf{u}_{o}=\tilde{\nabla} p_{o}, \partial p_{o} / \partial z=0, \\
\tilde{\boldsymbol{\nabla}} \cdot \mathbf{u}_{s}+\partial w_{s} / \partial z-0 \\
-\widetilde{\boldsymbol{\nabla}} p_{s}+\partial^{2} \mathbf{u}_{s} / \partial z^{2}-\left(\overline{\mathbf{u}_{o}} \cdot \widetilde{\boldsymbol{\nabla}}\right) \mathbf{u}_{o}+\bar{w}_{o} \partial \mathbf{u}_{o} / \partial z+\text { c.c. } \\
\partial p_{s} / \partial z=0
\end{gathered}
$$

if $(x, y) \in \Omega$ and $f_{s}<z<1$, and

$$
\begin{gathered}
\mathbf{u}_{s}-\mathbf{0}, w_{o}-w_{s}-0 \text { if } z-1, \\
w_{o}-i f_{o}+\mathbf{u}_{o} \cdot \widetilde{\mathbf{v}} f_{s}, \quad w_{s}-\partial f_{s} / \partial \hat{t}+\mathbf{u}_{s} \cdot \widetilde{\mathbf{\nabla}} f_{s}, \\
\partial \mathbf{u}_{s} / \partial z=\mathbf{0} \text { if } z=f_{s}, \\
p_{o}-\int_{s} / 2, p_{s}-\left(\int_{o}+f_{o}\right) / 2+\hat{B} \hat{C}^{-2} f_{s}+\hat{C}^{-2} \widetilde{\Delta} f_{s} \text { if } z-f_{s},
\end{gathered}
$$

$$
\int_{\Omega} f_{s} d \tilde{x} d \tilde{y}=0
$$

Note that viscous terms have been ignored (because they are small compared to inertia) in the second equation (3.3). This approximation fails in two thin viscous boundary layers, with $O\left(\omega^{1 / 2}\right)$ thicknesses, attached to the free surface and the upper plate; but the effect of these [which could in principle change the boundary conditions (3.5)-(3.7)] is seen to be of higher order and thus can be ignored in first approximation in both the oscillatory and the nonoscillatory parts of the solution. Inertia is much smaller in the second equation (3.4), where viscous terms cannot be neglected because they are of the same order as the convective terms. And, as in Sec. II, the effect of the lateral walls is appreciated only in a lateral boundary layer, with a $O(1)$ thickness, near the lateral walls where Eqs. (3.3)-(3.7) do not apply. This boundary layer (see Appendix A) provides the following boundary conditions for the solution in the bulk

$$
\partial f_{s} / \partial \tilde{n}--\hat{b} \partial f_{s} / \partial \hat{t} \text { or } f_{s}-0, \int_{0}^{1} \mathbf{u}_{s} \cdot \tilde{\mathbf{n}} d z-0 \text { on } \partial \widetilde{\Omega}
$$

On the other hand, we consider the following overall continuity equations, which are obtained upon integration of the first cxpressions in Eqs. (3.3) and (3.4) in $f_{s}<z<1$ and substitution of the first two boundary conditions (3.6),

$$
\tilde{\mathbf{\nabla}} \cdot\left(\int_{f_{s}^{\prime}}^{1} \mathbf{u}_{o} d z\right)=i f_{o}, \tilde{\mathbf{\nabla}} \cdot\left(\int_{f_{s}}^{1} \mathbf{u}_{s} d z\right)=\partial f_{s} / \partial \hat{t} .
$$

Using these, we may integrate the remaining equations and boundary conditions in Eqs. (3.3)-(3.7) to oblain

$$
p_{o}-f_{s} / 2, \quad \mathbf{u}_{o}-i \widetilde{\nabla} f_{s} / 2, \quad f_{o}-\left(\mathbf{1}-\int_{s}\right) \tilde{\Delta} \int_{s} / 2-\left|\widetilde{\nabla} f_{s}\right|^{2} / 2
$$




$$
\begin{gathered}
\mathbf{u}_{s}-\left(z^{2}-2 f_{s} z-1+2 f_{s}\right)\left[4 \widetilde{\nabla} p_{s}+\tilde{\nabla}\left(\left|\widetilde{\nabla} f_{s}\right|^{2}\right)\right] / 8, \\
\partial f_{s} / \partial \hat{t}--\tilde{\nabla} \cdot\left[\left(1-f_{s}\right)^{3} \tilde{\nabla}\left[4 p_{s}+\left|\tilde{\nabla} f_{s}\right|^{2}\right)\right] / 12, \\
p_{s}-\hat{B} C^{-2} f_{s}+\left[C^{-2}+\left(1-f_{s}\right) / 2\right] \widetilde{\Delta} f_{s}-\left|\tilde{\nabla} f_{s}\right|^{2} / 2 \text { in } \widetilde{\Omega},
\end{gathered}
$$

where we have taken into account that $\left(\widetilde{\nabla} f_{s}, \widetilde{\nabla}\right) \widetilde{\nabla} f_{s}$ $=\widetilde{\nabla}\left(\left|\widetilde{\nabla} f_{s}\right|^{2}\right) / 2$.

The evolution equation we were looking for is given by Eqs. (3.11) and (3.12). Also, invoking Eqs. (3.8), (3.9), and (3.10), we have

$$
\begin{gathered}
\partial f_{s} / \partial \tilde{n}--\hat{D} \partial f_{s} / \partial \hat{t} \text { or } f_{s}-0 \\
4 \partial p_{s} / \partial \tilde{n} \mid \partial\left(\left|\widetilde{\nabla} f_{s}\right|^{2}\right) / \partial \tilde{n}=0 \text { on } \partial \tilde{\Omega}, \quad \int_{\tilde{\Omega}} f_{s} d \tilde{x} d \tilde{y}=0
\end{gathered}
$$

And for convenience we rescale the time variable and drop out the subscript $s$ to rewrite Eqs. (3.11)-(3.13) as

$$
\begin{gathered}
\partial f / \partial \tau^{-}-\tilde{\nabla} \cdot\left[(1-f)^{3} \tilde{\nabla} l\right], \text { with } \\
y-\lambda f+\left(1-\gamma f \tilde{\Delta} f-\gamma|\tilde{\nabla} f|^{2} / 2, \text { in } \widetilde{\Omega},\right. \\
\partial f / \partial \tilde{n}--\beta \partial f / \partial \tau \text { or } f-0, \quad \partial l / \partial \tilde{n}-0 \text { on } \partial \widetilde{\Omega}, \\
\int_{\tilde{\Omega}} \int d \tilde{x} d \tilde{y}-0,
\end{gathered}
$$

where [see also Eqs. (1.13) and (3.2)]

$$
\begin{gathered}
\gamma-2 \hat{B} /\left(2+\hat{C}^{2}\right) \equiv 2 B L^{2} /\left(2+a^{2} \omega^{2} C^{2}\right) \\
\gamma-\hat{C}^{2} /\left(2+\hat{C}^{2}\right) \equiv a^{2} \omega^{2} C^{2} /\left(2+a^{2} \omega^{2} C^{2}\right)<1 \\
\beta-\left(2+\hat{C}^{2}\right) \hat{D} /\left(6 \hat{C}^{2}\right) \equiv D /\left(3 C^{2} / l^{3}\right) \\
\tau^{-}\left(2+\hat{C}^{2}\right) \hat{l} /\left(6 C^{2}\right) \equiv\left(2+a^{2} \omega^{2} C^{2}\right) l /\left(6 C^{2} l^{4}\right) .
\end{gathered}
$$

Equation (3.14) is somewhat similar to the Cahn-Hilliard equation. Since $0<\gamma<1$, the problem (3.14) is uniformly parabolic and thus has a unique solution satisfying given initial conditions $[19-21]$ whenever

$$
|f| \text {-bounded and } f<1 \text {. }
$$

Note that the first boundary condition is somewhat nonstandard, but it is dissipative because $\beta \geqslant 0$ and thus standard results for Dirichlet and Neumann boundary conditions are somewhat straightforwardly extended when this condition applies. In addition to the solutions satisfying Eq. (3.17) for all $\tau>0$, we could allow $f-1$ in a time-dependent closed subset $K(\tau) \subset \widetilde{\Omega}$, which phy sically corresponds to a dry spot on the upper plate. The associated problem, not considered here, would be a free boundary problem and should be completed with appropriate jump conditions at the boundary of K.

\section{B. Lincar stability of the flat state}

The linear stability of the simplest steady state of Eqs. (3.14) and (3.15), $f-0$, is analyzed as usually, by first linearizing around $f=0$ and then replacing $f(\tilde{x}, \tilde{y}, \tau)$ by $F(\tilde{x}, \tilde{y}) e^{\mu \tau}$ in the resulting problem, to obtain the linear eigenvalue problem

$$
\begin{gathered}
-\tilde{\Delta} U-\mu F, \quad \tilde{\Delta} F+\lambda F-U \text { in } \tilde{\Omega}, \\
\partial F / \partial \tilde{n}--\mu \beta F \text { or } F-0, \quad \partial U / \partial \tilde{n}-0 \text { on } \partial \tilde{\Omega}, \\
\int_{\tilde{\Omega}} F d \tilde{x} d \tilde{y}-0,
\end{gathered}
$$

which is analyzed in Appendix B. According to Property B1 the instability threshold is $\lambda-\lambda_{0}$ and invoking the first $\mathrm{ex}$ pression in Eq. (3.16), the main result in Sec. II [namely, Eq. $(2.40)]$ is recovered.

\section{Nonflat steady states without dry spots}

The steady states of Eqs. (3.14) and (3.15) that do not exhibit dry spots are given by

$$
\begin{aligned}
& (1-\gamma f) \tilde{\Delta} f+\lambda f-\gamma|\tilde{\nabla} f|^{2} / 2-\text { const, } f<1 \quad \text { in } \widetilde{\Omega}, \\
& \partial f / \partial \tilde{n}=0 \text { or } f=0 \text { on } \partial \widetilde{\Omega}, \quad \int_{\tilde{\Omega}} f d \tilde{x} d \tilde{y}=0 .
\end{aligned}
$$

As seen in Sec. III B above, the flat steady state $f-0$ is stable if $\lambda<\lambda_{0}$. Since Eqs. (3.20) and (3.21) are a particular case of Eqs. (C1) and (C2), with

$$
H(f)=\gamma f
$$

we may apply the analysis in Appendix $\mathrm{C}$ to obtain the following property concerning the local bifurcation of Eqs. (3.20) and (3.21) at $\lambda=0$.

Property 3.1. For generic domains $\widetilde{\Omega}$, such that the eigenfunctions of Eq. (2.14) associated with $\lambda-\lambda_{0}$, are such that

$$
\int_{\tilde{\Omega}} H_{0}^{3} d \tilde{x} d \tilde{y} \neq 0,
$$

the bifurcation is transcritical. And if $\widetilde{\Omega}$ is either a circle or a rectangle, then the bifurcation is subcritical.

Proof. Since $/ I^{\prime}(0)-\gamma>0$, if Eq. (3.23) holds, then the constant $\Gamma_{2}$ in Eq. ( $\left.\mathrm{C} 12\right)$ is nonzero and according to the discussion in Appendix $\mathrm{C}$, the bifurcation is Iranscritical. And since $H^{\prime \prime}(0)=0$, Property $\mathrm{C} 1$ implies that for circles and rectangles the bifurcation is subcritical.

The following global result gives sufficient conditions for nonexistence of nonflat stcady states without dry spots. 
Property 3.2. Let $\lambda_{0}>0$ be the lowest positive eigenvalue of Eq. (2.14). If $\gamma<2 / 3$ and $\lambda<\lambda_{0}(1-3 \gamma / 2)$ then Eqs. (3.20) and (3.21) only possesses the fat solution $f-0$.

Proof. In order to prove this property, we first note that the solutions of Eqs. (3.20) and (3.21) satisfy

$$
\int_{\tilde{\Omega}}\left[(1-3 \gamma f / 2)|\tilde{\nabla} f|^{2}-\lambda f^{2}\right] d \tilde{x} d \tilde{y}-0
$$

as readily obtaincd upon multiplication of Eq. (3.20) by $f$, integration in $\widetilde{\Omega}$, integration by parts and substitution of Eq. (3.21). And we only need to use the variational definition (B10) of $\lambda_{0}$ to obtain the stated result.

D. Lyapunov function and large-time behavior

The problem (3.14) and (3.15) admits a Lyapunov function that is readily obtained upon multiplication of the first equation (3.14) by $U$, integration in $\widetilde{\Omega}$, substitution of the second equation (3.14) and of Eq. (3.15), and integration by parts, to obtain

$$
\begin{array}{r}
d \mathcal{E} / d \tau^{-}-\int_{\tilde{\Omega}}\left(1-\rho^{3}|\tilde{\nabla} U|^{2} d \tilde{x} d \tilde{y}\right. \\
-\beta \int_{\partial \tilde{\Omega}}(1-\gamma f)(\partial f / \partial \tau)^{2} d s \\
\text { or } d \mathcal{E} / d \tau^{-}-\int_{\tilde{\Omega}}(1-f)^{3}|\tilde{\nabla} U|^{2} d \tilde{x} d \tilde{y}
\end{array}
$$

depending on whether the first or the second boundary condition (3.15) applies, where the rescaled energy $\mathcal{E}$ is given by

$$
\mathcal{E}^{-} \int_{\tilde{\Omega}}\left[(1-\gamma f) \mid \tilde{\nabla} f^{2}-\lambda f^{2}\right] d \tilde{x} d \tilde{y} / 2 .
$$

Equation (3.25) and a well-known result on infinitedimensional dynamical systems (Ref. [22]. p. 50, Lemma 3.8.2) (whose assumptions are checked in this case by embedding theorems [23] and a priori estimates for elliptic $[24,25]$ and parabolic $[19]$ equations) yields the following.

Property 3.3. If a solution of Eqs. (3.14) and (3.15) satisfies Eq. (3.17), uniformly for all $\tau>0$, then $f$ converges to a the set of steady states without dry spots as $\tau-\infty$.

As a consequence of this property, each solution of Eqs. (3.14) and (3.15) is such that either (i) becomes unbounded or develops a dry spot (for finite or infinite time) or (ii) converges to the set of steady states without dry spots, considered above in Secs. III B and III C.

\section{TWO IMMISCIBLE LAYERS}

We consider now a closed container of height $2 h$ and width $\mathscr{C}$ such that $h \ll \mathscr{C}$, which is filled with two immiscible liquids of different densities, with the lighter liquid below the heavier one. We use a vibrating Cartesian coordinate system with the $z^{-0}$ plane on the unperturbed interface, assumed to be horizontal, and employ the viscous time $h^{2}\left(\rho^{+}+\rho^{-}\right) /\left(\rho^{+} \nu^{+}+\rho^{-} \nu^{-}\right)$and the length $h$ for nondi- mensionalization, where $\rho$ and $\nu$ denote the density and kinematic viscosity and the superscripts + and - are used hereinafter for the variables pertaining to the liquid above and below the interface. The governing equations are now

$$
\begin{gathered}
\boldsymbol{\nabla} \cdot \mathbf{u}^{+}+\partial w^{-} / \partial z-0, \\
(1 \pm m)\left[\partial \mathbf{u}^{+} / \partial t+\left(\mathbf{u}^{-} \cdot \nabla\right) \mathbf{u}^{+}+w^{+} \partial \mathbf{u}^{-} / \partial z\right] \\
=2 \nabla p^{ \pm} \mid(1 \pm n)\left(\Delta \mathbf{u}^{ \pm} \mid \partial^{2} \mathbf{u}^{ \pm} / \partial z^{2}\right), \\
(1 \pm m)\left[\partial w^{ \pm} / \partial t\left|\mathbf{u}^{ \pm} \cdot \nabla w^{ \pm}\right| w^{ \pm} \partial w^{ \pm} / \partial z\right] \\
--2 \partial p^{\perp} / \partial z+(1, n)\left(\Delta w^{\perp}+\partial^{2} w^{\perp} / \partial z^{2}\right),
\end{gathered}
$$

if $(x, y) \in \Omega$ and $|f(x, y, t)<| z<1 \mid \delta$, with boundary conditions

$$
\begin{aligned}
& \mathbf{u}^{+}-\mathbf{0}, w^{+}-0 \text { if } z-\prime^{\prime}\left(1^{\prime} \delta\right) \text { and if }(x, y) \in \partial \Omega \text {. } \\
& \mathbf{u}-\mathbf{u}^{\prime}, w-\mathbf{u} \cdot \boldsymbol{\nabla} f-w^{\prime}-\mathbf{u}^{\prime} \cdot \boldsymbol{\nabla} f-\partial f / \partial t \text { if } z-f \text {, } \\
& (1 \mid n)\left(\partial \mathbf{u} / \partial z \mid \nabla w^{\prime}\right) \quad(1 \quad n)(\partial \mathbf{u} / \partial z \mid \nabla w) \\
& -O\left(\left|\boldsymbol{\nabla} \mathbf{u}^{+}\right||\boldsymbol{\nabla} f|+\left(\left|\partial \mathbf{u}^{+} / \partial z\right|+\left|\boldsymbol{\nabla}_{w^{+}}\right|\right)|\boldsymbol{\nabla} f|^{2}\right) \text { if } z-f, \\
& p^{-}-p^{-}-a \omega^{2} f \cos (\omega t)-B C^{-2} f \\
& -C^{-2} \boldsymbol{\nabla} \cdot\left[\boldsymbol{\nabla} f /\left(\mathbf{1}+|\boldsymbol{\nabla} /|^{2}\right)^{1 / 2}\right] \\
& -2(1+n) \partial w^{1} / \partial z-2(1-n) \partial w / \partial z \\
& +O\left(\left|\boldsymbol{\nabla} \mathbf{u}^{+}\right|+\left(\left|\partial \mathbf{u}^{+} / \partial z\right|+\left|\boldsymbol{\nabla} w^{+}\right|\right)|\boldsymbol{\nabla} f|\right) \text { if } z^{-} f,
\end{aligned}
$$

$$
\begin{gathered}
\boldsymbol{\nabla} f \cdot \mathbf{n}--D \partial f / \partial t \text { or } f-0 \text { if }(x, y) \in \partial \Omega \\
\int_{\Omega} f(x, y, t) d x d y=0
\end{gathered}
$$

where $\mathbf{u}, w, p, f, \boldsymbol{\nabla}, \boldsymbol{\nabla} \cdot \Delta, \Omega, \partial \Omega$, and $\mathbf{n}$ are as defined in Sec. I, with $f$ and $L$ (the dimensionless characteristic size of $\Omega$ ) satisfying again Eqs. (1.8) and (1.9). The positive parameters $\delta, m$ (Atwood number) and $n$, the effective nondimensional vibration amplitude, $a$, and the Bond and capillary numbers are now

$$
\begin{gathered}
\delta-\left(h^{-}-h^{-}\right) /\left(h^{+}+h^{-}\right), m-\left(\rho^{+}-\rho^{-}\right) /\left(\rho^{-}+\rho^{-}\right), \\
n=\left(\rho^{\prime} \nu \cdot \quad \rho \nu\right) /\left(\rho^{\cdot} \nu^{\prime} \mid \rho \nu\right), \\
a-\left(a^{*} / h\right)\left(\rho^{+}-\rho^{-}\right) /\left(\rho^{+}+\rho^{-}\right) \\
B-\left(\rho^{+}-\rho^{-}\right) g h^{2} / \sigma>0
\end{gathered}
$$

and

$$
C^{-}\left(\rho^{+} \nu^{+}+\rho^{-} \nu^{-}\right)\left[\left(\rho^{-}+\rho^{-}\right) \sigma h\right]^{-1 / 2},
$$


where $a^{*}$ is the dimensional vibrating amplitude, $h^{\perp}$ are the unperturbed depths of the liquid layers (such that $h^{+}+h^{-}$ $-2 h), p^{+}$are the densitics, $g$ is the gravitational acceleration, $\nu^{ \pm}$are the kinematic viscosities, and $\sigma$ is the surface tension coeflicient.

In the absence of vibration (if $a-0$ ) the quiescent state is linearly unstable due to RT instability, if and only if, Eq. (2.13) holds. With vibration, the lincar stability analysis is completely similar to that in Sec. II and yields the asy mptotic stability condition

$$
\hat{\beta}<\hat{B}_{c}-\lambda_{0}\left[1+\left(1-\delta^{2}\right) a^{2} \omega^{2} C^{2} /\{2(1-m \delta)\}\right],
$$

where $\hat{B}=B L^{2}$ is as defined in Eq. (2.13), $\lambda_{0}$ is the lowest eigenvalue of Eq. (2.14) and, as in Sec. II, we assume that

$$
\omega \gg 1 \text {. }
$$

With the same notation as in Sec. II B, remark (3), condition (4.10) can be written in dimensional form as

$$
\begin{aligned}
\ell< & \lambda_{61}\left[\frac{\sigma}{\left(\rho^{-}-\rho^{-}\right) g} \mid \frac{\left(2 \pi a^{*} \omega^{*}\right)^{2}}{2 g}\right. \\
& \left.\times \frac{h^{\prime} h\left(\rho^{\prime}+\rho\right)^{2}}{\left(h^{-} \rho^{+}+h^{+} \rho^{-}\right)\left(\rho^{-}-\rho^{-}\right)}\right] .
\end{aligned}
$$

Note that as in the case of only one liquid layer and for the same reason [remark (3), at the end of Scc. II B] this condition is independent of viscosity.

In the limit (4.11), the analysis of the weakly nonlinear dynamics of the system proceeds as in Sec. III. We again assume that $\mathrm{Eq}$. (3.1) holds, rescale $C, D$, and the slow time variable as

$\hat{C}-a \omega C, \quad \hat{D}-2 a^{2} \omega^{2} D /\left[\left(2+a^{2} \omega^{2} C^{2}\right) L^{3}\right], \quad \hat{t}-a^{2} \omega^{2} t / L^{4}$,

where $\hat{C}$ and $\hat{D}$ are again assumed of order unity, and seek the expansions

$$
\begin{aligned}
& \mathbf{u}^{ \pm}=a \omega L^{-1} \mathbf{u}_{\theta}^{\perp}(\tilde{x}, \tilde{y}, z, \hat{t}) e^{i \omega t} \mid a^{2} \omega L^{-3} \text { h.o.h. I c.c. } \\
& \left|a^{2} \omega^{2} L{ }^{3} \mathbf{u}_{s}^{-}(\tilde{x}, \tilde{y}, z, \hat{t})\right| \cdots, \\
& w^{+}-a \omega L^{-2} w_{o}=(\tilde{x}, \tilde{y}, z, \hat{t}) e^{i \omega t}+a^{2} \omega L^{-4} \text { h.o.h. }+ \text { c.c. } \\
& +a^{2} \omega^{2} l^{-4} w_{s}^{ \pm}(\tilde{x}, \tilde{y}, z, \hat{\imath})+\cdots, \\
& p^{+}-a \omega^{2} p_{o}^{ \pm}(\tilde{x}, \tilde{y}, z, i) e^{i \omega t}+a^{2} \omega^{2} l^{-2} \text { h.o.h. }+ \text { c.c. } \\
& +a^{2} \omega^{2} l^{-2} p_{s}^{\frac{1}{(}}(\tilde{x}, \tilde{y}, z, \hat{\imath})+\cdots,
\end{aligned}
$$

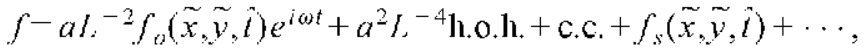

which are now replaced into the original nonlinear problem (4.1)-(4.8), to oblain

$$
\partial p_{o}^{+} / \partial z-0, \quad i(1+m) \mathbf{u}_{o}^{-}--2 \widetilde{\nabla} p_{o}^{-}, \quad \widetilde{\nabla} \cdot \mathbf{u}_{o}^{+}+\partial w_{o}^{+} / \partial z-0,
$$

$$
\partial p_{s}^{-} / \partial z-0
$$

$$
\begin{aligned}
(1 \pm n) \partial^{2} \mathbf{u}_{s}^{\frac{1}{2}} / \partial z^{2}- & 2 \widetilde{\nabla} p_{s}^{\dot{-}}+(1 \pm m) \\
& \times\left[\left(\overline{\mathbf{u}_{o}^{\perp}} \cdot \widetilde{\nabla}\right) \mathbf{u}_{o}^{\perp}+w_{o}^{\frac{1}{\partial}} \partial \mathbf{u}_{o}^{-} / \partial z+\text { c.c. }\right] \\
& \tilde{\nabla} \cdot \mathbf{u}_{s}^{\perp}+\partial w_{s}^{\frac{1}{2}} / \partial z-0
\end{aligned}
$$

in $(\tilde{x}, \tilde{y}) \in \widetilde{\Omega}, \pm f_{s}< \pm z<1 \pm \delta$, and

$$
\begin{gathered}
\mathbf{u}_{s}^{\perp}=\mathbf{0}, w_{o}^{\perp}=w_{s}^{\perp}=0 \text { if } z= \pm(1 \pm \delta), \\
w_{o}^{\perp} \quad \mathbf{u}_{o}^{-} \cdot \widetilde{\nabla} f_{s}=i f_{o}, w_{s}^{\perp} \quad \mathbf{u}_{s}^{\perp} \cdot \widetilde{\nabla} f_{s}=\partial f_{s} / \partial \hat{t} \text { if } z=f_{s}, \\
\mathbf{u}_{s}^{-}-\mathbf{u}_{s}^{-},(1-n) \partial \mathbf{u}_{s}^{-} / \partial z-(1+n) \partial \mathbf{u}_{s}^{+} / \partial z \text { if } z-f_{s},
\end{gathered}
$$

$$
\begin{aligned}
& p_{o}^{\prime}-p_{o}-f_{s} / 2, \\
& p_{s}^{+}-p_{s}^{-}-\left(f_{o}+\overline{f_{o}}\right) / 2+\hat{B} \hat{C}^{-2} f_{s}+\hat{C}^{-2} \widetilde{\Delta} f_{s} \text { if } z-f_{s} .
\end{aligned}
$$

And again, the analysis of the lateral boundary layer near the lateral wall (see Appendix A) and volume conservation yield

$$
\begin{gathered}
\partial f_{s} / \partial \tilde{n}=\hat{D} \partial f_{s} / \partial \hat{t} \text { or } f_{s}=0, \\
\int_{-(1-\delta)}^{f_{s}} \mathbf{u}_{s}^{-} \cdot \tilde{\mathbf{n}} d z-\int_{f_{s}}^{(1+\delta)} \mathbf{u}_{s}^{-} \cdot \tilde{\mathbf{n}} d z-0, \\
\int_{-(1-\delta)}^{f_{s}} \mathbf{u}_{o}^{-} \cdot \tilde{\mathbf{n}} d z \mid \int_{f_{s}}^{(1 \mid \delta)} \mathbf{u}_{o}^{-} \cdot \tilde{\mathbf{n}} d z=0 \text { on } \partial \tilde{\mathbf{\Omega}}, \\
\int_{\tilde{\Omega}} f_{s} d \tilde{x} d \tilde{y}-0 .
\end{gathered}
$$

As in Sec. III A, the following overall continuity equations in the lower and upper layers are useful

$$
\tilde{\boldsymbol{\nabla}} \cdot\left(\int_{f_{s}}^{\dot{(1}(1 \perp \delta)} \mathbf{u}_{o}^{-} d z\right)-i f_{o}, \tilde{\boldsymbol{\nabla}} \cdot\left(\int_{f_{s}}^{\perp(1-\delta)} \mathbf{u}_{s}^{+} d z\right)-\partial f_{s} / \partial \hat{t}
$$

which follow upon integration of the last expressions in Eqs. (4.13) and (4.14) in $\pm(1 \pm \delta)< \pm z< \pm f_{x}$ and substitution of Eq. (4.16). Using the first of these, the oscillatory problem, posed by Eq. (4.13), with boundary conditions (4.15), (4.16), (4.18), and (4.19), is readily integrated to oblain

$$
\begin{gathered}
p_{o}^{\perp}=\left(g_{o} \pm f_{s}\right) / 4, \quad \mathbf{u}_{o}=2 i(1 \pm m)^{-1} \widetilde{\nabla} p_{o}^{\perp}, \\
f_{o}-(1+m)^{-1} \widetilde{\nabla} \cdot\left[\left(1+\delta-f_{s}\right) \widetilde{\nabla}\left(g_{0}+f_{s}\right) / 2\right],
\end{gathered}
$$

where $g_{0}$ is uniquely given by 


$$
\begin{aligned}
& \widetilde{\boldsymbol{\nabla}} \cdot\left[\left(1+m f_{s}-m \delta\right) \widetilde{\nabla} g_{0}+\left(\delta-f_{s}-m\right) \tilde{\nabla} f_{s}\right]-0 \text { in } \widetilde{\Omega} \text {, } \\
& {\left[\left(1 \mid m f_{s} \quad m \delta\right) \tilde{\nabla} g_{0} \mid\left(\begin{array}{lll}
\delta & f_{s} & m
\end{array}\right) \tilde{\nabla} f_{s}\right] \cdot \tilde{\mathbf{n}}=0 \text { on } \partial \widetilde{\Omega} .}
\end{aligned}
$$

Here the expression between brackets exactly coincides with $\int_{-(1-\delta)}^{f_{s}} \mathbf{u}_{o}^{-} d z+\int_{f_{s}}^{1+\delta_{s}} \mathbf{u}_{o}^{-} d z$. This linear problem is readily solved to obtain

$$
\begin{gathered}
g_{0}(\tilde{x}, \tilde{y}, \hat{t})-G_{0}\left(f_{s}(\tilde{x}, \tilde{y}, \hat{t})\right), \text { with } \\
G_{0}\left(f_{s}\right)-\int_{0}^{f_{s}}[(\xi+m-\delta) /(\mathbf{1}+m \xi-m \delta)] d \xi
\end{gathered}
$$

In order to avoid too involved expressions, we do not consider the most general values of the parameters in the sequel, but only two limiting cases that bear the main ingredients of the general case. These two cases are that in which the viscosities of the liquids are disparate [i.e., $n-1$ or

1, see Eq. (4.9)] and that in which the viscosities, densities and unperturbed depths of both layers are approximately the samc.

\section{A. Disparate viscositics but arbitrary Atwood number and unperturbed depths}

Without loss of generality we assume that

$$
\rho^{+} \nu^{+} \geqq \rho^{-} \nu^{-} \text {. }
$$

Then $n=1$ and using Eqs. (4.14)-(4.20), the nonoscillatory flow is readily obtained to be

$$
\begin{aligned}
& p_{s}=2\left(\begin{array}{ll}
1 & m
\end{array}\right)^{-1}\left|\widetilde{\nabla} p_{o}\right|^{2}, \\
& p_{s}^{+}=2(1 \mathrm{~m}){ }^{1}\left|\widetilde{\nabla} p_{o}^{-}\right|^{2}\left|f_{0}\right| \hat{B} \hat{C}^{2} f_{s} \mid \hat{C}{ }^{2} \widetilde{\Delta} f_{s}, \\
& \mathbf{u}_{s}^{\prime}=\left[\widetilde{\mathbf{v}}_{p_{s}} \cdot \mid 2(1 \mid m)^{-1} \widetilde{\mathbf{\nabla}}\left(\left|\widetilde{\mathbf{v}} p_{0}^{\prime}\right|^{2}\right)\right] \\
& \left.\times\left[\begin{array}{ll}
z & f_{s}
\end{array}\right)^{2} \quad\left(\begin{array}{llll}
1 & \mid & \delta & f_{s}
\end{array}\right)^{2}\right] / 2,
\end{aligned}
$$$$
\partial f_{s} / \partial \hat{t}--\widetilde{\mathbf{\nabla}} \cdot\left(\left(1+\delta-f_{s}\right)^{3} \widetilde{\mathbf{\nabla}}\left[p_{s}^{+}+2(1+m)^{-1}\left|\widetilde{\mathbf{\nabla}} p_{o}^{+}\right|^{2}\right]\right) / 3,
$$

where we have laken into account the vector identity $\left(\widetilde{\mathbf{\nabla}} p_{o}^{+}, \widetilde{\nabla}\right) \widetilde{\nabla} p_{o}^{+}=\tilde{\mathbf{\nabla}}\left(\left|\widetilde{\nabla} p_{o}^{-}\right|^{2}\right) / 2$. Thus $f_{s}$ evolves according to the parabolic equation (4.26) where, according to Eqs. (4.21), (4.22) and (4.25), $p_{s}^{\prime}+2(1+m)^{-1}\left|\widetilde{\nabla_{p}} p_{o}^{\cdot}\right|^{2}$ is given by

$$
\begin{aligned}
2 p_{s}^{\prime} \mid & 4(1 \mid m)^{-1}\left|\widetilde{\nabla} p_{o}^{\prime}\right|^{2} \\
& =(1 \quad m)^{-1}\left|\widetilde{\nabla}\left(g_{0} \quad f_{s}\right) / 2\right|^{2}\left|(1 \mid m)^{-1}\right| \\
& \times \widetilde{\nabla}\left(g_{0}+f_{s}\right) /\left.2\right|^{2}+(1+m)^{-1} \widetilde{\nabla} \cdot\left[\left(1+\delta-f_{s}\right)\right. \\
& \left.\times \widetilde{\nabla}\left(g_{0} \mid f_{s}\right)\right]\left|2 \hat{B} \hat{C}^{-2} f_{s}\right| 2 \hat{C}^{-2} \widetilde{\Delta} f_{s},
\end{aligned}
$$

with $g_{0}$ as given by Eq. (4.23). In addition, we have

$$
\partial f_{s} / \partial \tilde{n}--\hat{D} \partial f_{s} / \partial \hat{l} \text { or } f_{s}-0
$$

$$
\begin{gathered}
\tilde{\mathbf{\nabla}}\left[p_{s}^{+}+2(1+m){ }^{1}\left|\tilde{\mathbf{\nabla}} p_{o}^{+}\right|^{2}\right] \cdot \tilde{\mathbf{n}}-0 \text { on } \tilde{\partial} \Omega, \\
\int_{\tilde{\Omega}} f_{s} d \tilde{x} d \tilde{y}=0
\end{gathered}
$$

which result from Eq. (4.19) when taking into account that

$$
\begin{aligned}
\int_{f_{s}^{\prime}}^{1} \mathbf{u}_{s}^{-} d z- & -\left[\widetilde{\mathbf{\nabla}} p_{s}^{-}+2(1+m){ }^{l} \widetilde{\mathbf{\nabla}}\left(\left|\widetilde{\mathbf{\nabla}} p_{o}^{+}\right|^{2}\right)\right] \\
& \times\left(1+\delta-f_{s}\right)^{3} / 3 .
\end{aligned}
$$

As in Sec. III, we rescalc $\hat{i}$ and drop out the subscript ss to rewrite Eqs. (4.26)-(4.28), after some algebra, as

$$
\begin{gathered}
\partial f / \partial \tau--\tilde{\nabla} \cdot\left[(1+\delta-f)^{3} \widetilde{\nabla} U\right], \text { with } \\
U-\lambda f+[1-l l(f)] \widetilde{\Delta} f-I I^{\prime}(\rho)|\widetilde{\nabla} f|^{2} / 2, \text { in } \widetilde{\Omega},
\end{gathered}
$$

$\partial f / \partial \tilde{n}--\beta \partial f / \partial \tau$ or $f-0, \quad \partial U / \partial \tilde{n}-0$ on $\partial \tilde{\Omega}$,

$$
\int_{\tilde{\Omega}} \int d \tilde{x} d \tilde{y}-0
$$

where the function $/ /$ is defined as

$$
H(f)=1 \frac{2+\hat{C}^{2}\left[1-(\delta-f)^{2}\right] /[1+m(f-\delta)]}{2+\hat{C}^{2}\left(1-\delta^{2}\right) /(1-m \delta)}
$$

and the parameters $\lambda$ and $\beta$ and the lime variable $\tau$ are given by

$$
\begin{aligned}
& \lambda-\frac{2 \hat{B}}{2+C^{2}\left(1-\delta^{2}\right) /(1-m \delta)} \\
& \equiv \frac{2 B L^{2}}{2+a^{2} \omega^{2} C^{2}\left(1-\delta^{2}\right) /(1-m \delta)},
\end{aligned}
$$

$$
\beta-\frac{\left(2+\hat{C}^{2}\right) \hat{D}}{6 \hat{C}^{2}} \equiv \frac{D}{3 C^{2} l^{3}}, \quad \tau^{-} \frac{\left(2+\hat{C}^{2}\right) \hat{t}}{6 \hat{C}^{2}} \equiv \frac{\left(2+a^{2} \omega^{2} C^{2}\right) t}{6 / I^{4} C^{2}}
$$

Note that $H(0)-0$ and that $1-H(f)>0$ if

$$
-(1-\delta)<\delta<1+\delta,
$$

that is, if the interface does not touch the lower and upper boundaries of the container. In this case, the problem (4.29) and $(4.30)$ is uniformly parabolic and possesses a unique solution.

If $\rho^{-}-0$ then the effect of the lighter liquid disappears and we must recover the results in Sec. III. And this is true because if $m-\delta-1$ (we are also requiring $h^{+}-h^{-}$because of the nondimensionalization above) then Eqs. (4.29)-(4.31) coincide with Eqs. (3.14) and (3.15). On the other hand. assumption (4.24) means that the inviscid liquid is the lighter 
one, namely that placed below. The opposite case is obtained, still under assumption (4.24), by changing the direction of gravity and interchanging the lower and upper liquids, which means according to Eq. (4.9), to change the signs of $m$ and $n$. Thus both possibilities are included in Eqs. (4.29) and (4.30) by just allowing $m$ to vary between 1 and 1 .

The analysis in Secs. IIIB-IIID is readily extended to Eqs. (4.29) and (4.30). In particular,

(1) If, in order to analyze the linear stability of the flat steady state $f=0$, we linearize Eqs. (4.29) and (4.30) around $f-0$ and replace $f(\tilde{x}, \tilde{y}, \tau)$ by $F(\tilde{x}, \tilde{y}) e^{\mu \tau}$ in the resulting problem, then we obtain again the linear eigenvalue problem (3.18)-(3.19). Thus the instability threshold is again $\lambda-\lambda_{0}$. which according to Eq. (4.32) yields the following threshold value of the Bond number

$$
B L^{2}<\hat{B}_{c}-\lambda_{0}\left[1+\left(1-\delta^{2}\right) a^{2} \omega^{2} C^{2} /\{2(1-m \delta)\}\right] .
$$

Thus the threshold value (4.10) is recovered.

(2) As in Sec. IIIC, the bifurcation from the flat state at $\lambda-\lambda_{0}$ is transcritical for generic cross-sections, such that Eq. (3.2.3) holds. And since, according to Eq. (4.31),

$$
H^{\prime \prime}(0)=\frac{2\left(1-m^{2}\right) a^{2} \omega^{2} C^{2}}{(1-m \delta)^{2}\left[2(1-m \delta)+a^{2} \omega^{2} C^{2}\left(1-\delta^{2}\right)\right]}>0,
$$

Property $\mathrm{Cl}$, in Appendix $\mathrm{C}$, implies that bifurcation is subcritical if the cross section is either a circle or a rectangle.

(3) As in Sec. III A, a rescaled overall mechanical energy equation is obtained upon multiplication of Eq (4.29) by $U$, integration in $\widetilde{\Omega}$, substitution of Eqs. (4.29) and (4.30), and integration by parts, as

$$
\begin{aligned}
& d \varepsilon / d \tau--\int_{\tilde{\Omega}}(1-f)^{3}|\widetilde{\nabla} U|^{2} d \tilde{x} d \tilde{y} \\
& -\beta \int_{\partial \tilde{\Omega}}[1-/ I(f)](\partial f / \partial \tau)^{2} d s \\
& \text { or } d \mathcal{E} / d \tau--\int_{\tilde{\Omega}}(1-f)^{3}|\widetilde{\nabla} U|^{2} d \tilde{x} d \tilde{y} \text {, }
\end{aligned}
$$

depending on whether the first or the second boundary condition (4.30) applies, where the rescaled energy $\mathcal{E}$ is given by

$$
\mathcal{E}=\int_{\tilde{\Omega}}\left(\left[\begin{array}{ll}
1 & H(f)
\end{array}\right]|\tilde{\nabla} f|^{2} \quad \lambda f^{2}\right) d \tilde{x} d \tilde{y} / 2
$$

Thus the problem (4.29) and (4.30) admits a Lyapunov function and, proceeding as in Sec. III D, we conclude that the solutions that satisfy Eq. (4.33) uniformly in $0<\tau<\infty$ converge to the set of steady states that satisfy Eq. (4.33).

\section{B. Zero Atwood number and equal viscosities and unperturbed depths}

Now we take

$$
m-n-\delta-0 .
$$

Then Eq. (4.23) yields $G(\xi)-\xi^{2} / 2$ and the expressions (4.21) and (4.22) for $p_{0}^{-}$and $f_{0}$ reduce to

$$
p_{o}^{ \pm}-f_{s}^{2} / 8 \pm f_{s} / 4, f_{0}-\tilde{\nabla} \cdot\left[\left(1-f_{s}^{2}\right) \widetilde{\nabla} \int_{s} / 2\right] .
$$

And we only need to use Eqs. (4.14)-(4.20) to obtain the nonoscillatory flow as

$$
\begin{aligned}
& \mathbf{u}_{s}^{+}= \frac{\left(z-f_{s}\right)^{2} \widetilde{\nabla}\left(g_{1} \pm G_{1}\right)}{4} \\
&-\frac{\left(z-f_{s}\right)\left[-2 f_{s} \tilde{\nabla} g_{1}+\left(1+f_{s}^{2}\right) \widetilde{\nabla} G_{1}\right]}{4} \\
&-\frac{\left(1-f_{s}^{2}\right)\left(\tilde{\nabla} g_{1}-f_{s} \widetilde{\nabla} G_{1}\right)}{4}, \\
& p_{s}^{+}=\left(g_{1} \pm\left(G_{1}\right) / 4 \quad 2\left|\widetilde{\nabla} p_{o}^{-}\right|^{2}, \quad \partial f_{s} / \partial \hat{t}=\tilde{\nabla} \cdot \mathbf{U}_{s}^{+},\right.
\end{aligned}
$$

where $G_{1}$ and $\mathbf{U}_{s}^{+}--\int_{+1}^{f_{s}} \mathbf{u}_{s}^{-} d z$ are given by

$$
\begin{aligned}
& \left.G_{1}=2 \hat{B} \hat{C}^{2} f_{s}\left|2 \hat{C} \quad{ }^{2} \widetilde{\Delta} f_{s}\right| f_{s}\left|\widetilde{\nabla} f_{s}\right|^{2} \mid \widetilde{\nabla} \cdot\left[\begin{array}{ll}
1 & f_{s}^{2}
\end{array}\right) \widetilde{\nabla} f_{s}\right] \text {, } \\
& \mathbf{U}_{s}^{\perp}=\frac{\left(11-f_{s}\right)^{3} \widetilde{\nabla}\left(g_{1}, G_{1}\right)}{12} \\
& -\frac{\left( \pm 1 f_{s}\right)^{2}\left[2 f_{s} \widetilde{\nabla} g_{1} \mid\left(1 \mid f_{s}^{2}\right) \widetilde{\nabla} G_{1}\right]}{8} \\
& -\frac{\left(1-f_{s}^{2}\right)\left(11-f_{s}\right)\left(\tilde{\nabla} g_{1}-f_{s} \tilde{\nabla} G_{1}\right)}{4} \text {. }
\end{aligned}
$$

In addition, according to Eq. (4.19) we have the following boundary conditions

$$
\begin{gathered}
\partial f_{s} l \partial \tilde{n}--\hat{D} \partial f_{s} l \partial \hat{t} \text { or } f_{s}-0, \quad \mathbf{U}_{s}^{-} \cdot \tilde{\mathbf{n}}-0 \text { on } \partial \widetilde{\mathbf{\Omega}} \\
\int_{\tilde{\Omega}} f_{s} d \tilde{x} d \tilde{y}=0
\end{gathered}
$$

and using Eq. (4.37) the boundary conditions $\tilde{\mathbf{U}}_{s}^{ \pm} \cdot \tilde{\mathbf{n}}-0$ are secn to be equivalent to

$$
\partial g_{1} / \partial \tilde{n}-\partial G_{1} / \partial \tilde{n}-0 \text { on } \partial \widetilde{\Omega} .
$$

On the other hand, according to Eq. (4.36), we have $\tilde{\nabla} \cdot\left(\mathbf{U}_{s}^{\cdot}-\mathbf{U}_{s}\right)-0$; using Eqs. (4.20), (4.37), and (4.39) we obtain after some algebra

$$
2 \widetilde{\Delta} g_{1}=\tilde{\nabla} \cdot\left[\left(3 f_{s} f_{s}^{\rho}\right) \tilde{\nabla} G_{1}\right] \text { in } \widetilde{\Omega}, 2 \partial g_{1} / \partial \tilde{n}=0 \text { on } \partial \widetilde{\Omega} \text {. }
$$

This equation uniquely provides $g_{1}$ (up to an additive constant) in terms of $f_{s}$ as

$$
g_{1}=\mathcal{G}\left(\tilde{\nabla} \cdot\left[\left(3 f_{s} f_{s}^{3}\right) \tilde{\nabla}\left(g_{1}\right]\right) / 2,\right.
$$


where $\mathcal{G}$ is the Green (integral) operator of $-\widetilde{\Delta}$ in $\widetilde{\Omega}$, with homogeneous Neumann boundary conditions, defined by Eq. (B4) [where $U-\mathcal{G}(F)]$ in Appendix B. Substitution of Eq. (4.40) into Eq. (4.37) yields, after some algebra,

$$
\begin{aligned}
\widetilde{\mathbf{\nabla}} \cdot \mathbf{U}_{s}^{+}- & -\widetilde{\mathbf{\nabla}} \cdot\left[\left(1-f_{s}^{2}\right)^{3} \widetilde{\nabla} G_{1}\right] / 24 \\
& -\left[\left(1-f_{s}^{2}\right) / 8\right] \widetilde{\mathbf{\nabla}} f_{s} \cdot \widetilde{\mathbf{\nabla}}\left[\mathcal{G}\left\{\left(\tilde{\mathbf{\nabla}} \cdot\left[\left(3 f_{s}-f_{s}^{3}\right) \widetilde{\nabla} G_{1}\right]\right\}\right]\right. \\
& -\left[\left(1-f_{s}^{2}\right)\left(3 f_{s}-f_{s}^{3}\right) / 8\right] \widetilde{\mathbf{\nabla}} f_{s} \cdot \widetilde{\nabla} G_{1},
\end{aligned}
$$

where we have taken into account that $\Delta(G / /) \equiv f$ by definition of $\mathcal{G}$. And $f_{s}$ is given by the parabolic (integrodifferential) equation defined by the sccond equation in Eqs. (4.36) and (4.41), with the boundary conditions (4.38) and (4.39) and appropriate initial conditions. For convenience, this problem is rescaled as

$$
\begin{gathered}
\partial f / \partial \tau^{-}-\tilde{\nabla} \cdot\left[\left(1-f^{2}\right)^{3} \tilde{\nabla} U\right] / 4-\left[3\left(1-f^{2}\right) / 4\right] \\
\tilde{\nabla} f \cdot \tilde{\nabla}\left[\mathcal{G}\left(\tilde{\nabla} \cdot\left[\left(3 f-f^{3}\right) \tilde{\nabla} U\right]\right)\right] \\
-3\left(1-f^{2}\right)\left(3 f-f^{3}\right) \tilde{\nabla} f \cdot \tilde{\nabla} U / 4
\end{gathered}
$$

with

$$
\begin{aligned}
& U=\left.\lambda f\left|\left(1 \quad \gamma f^{2}\right) \widetilde{\Delta f} \quad \gamma f\right| \widetilde{\nabla} f\right|^{2}, \\
& \partial f / \partial \tilde{n}--\beta \partial f / \partial \tau \text { or } f-0, \partial U / \partial \tilde{n}-0 \text { on } \partial \widetilde{\Omega} \text {, } \\
& \int_{\tilde{\Omega}} f d \tilde{x} d \tilde{y}=0
\end{aligned}
$$

where we have dropped out the subscript $s$ from $f_{s}$ and the parameters $\gamma, \beta$, and $\lambda$, and the rescaled time variable $\tau$ are defined as [see also Eqs. (4.10) and (4.12)]

$$
\begin{gathered}
\lambda-2 \hat{B} /\left(2+\hat{C}^{2}\right) \equiv 2 B L^{2} /\left(2+a^{2} \omega^{2} C^{2}\right), \\
\gamma=\hat{C}^{2} /\left(2+\hat{C}^{2}\right) \equiv a^{2} \omega^{2} C^{2} /\left(2 \mid a^{2} \omega^{2} C^{2}\right)<1, \\
\beta^{-}\left(2+\hat{C}^{2}\right) \hat{D} /\left(6 \hat{C}^{2}\right) \equiv D /\left(3 C^{2} l^{3}\right), \\
\tau-\left(2+\hat{C}^{2}\right) \hat{t} /\left(6 \hat{C}^{2}\right) \equiv\left(2+a^{2} \omega^{2} C^{2}\right) t /\left(6 L^{4} C^{2}\right) .
\end{gathered}
$$

The analysis in Secs. III B-D and some of the results there are extended to Eqs. (4.42) and (4.43). The following comments are in order

(A) If, in order to analyze the linear stability of the flat steady state $f-0$, we linearize Eqs. (4.42) and (4.43) around $f^{-0}$ and replace $f(\tilde{x}, \tilde{y}, \tau)$ by $F^{\prime}(\tilde{x}, \tilde{y}) e^{\mu \tau}$ in the resulting problem, then we obtain again the linear eigenvalue problem (3.18) and (3.19). Thus the instability threshold is again $\lambda$ $=\lambda_{t i}$, which according to Eq. (4.44) yields the following threshold value of the Bond number

$$
B L^{2}<\hat{B}_{c}-\lambda_{01}\left(1+a^{2} \omega^{2} C^{2} / 2\right) .
$$

Thus we recover Eq. (4.10).
(B) When analyzing the bifurcation from the flat state at $\lambda=\lambda_{0}$ we may take $\partial f / \partial \tau=0, U=$ const in Eqs. (4.42) and (4.43), to obtain the problem (C1) and (C2) considered in Appendix C, with

$$
H(f)-\gamma f^{2}
$$

Thus the bifurcation is transcritical for generic cross sections, such that Eq. (3.23) holds. And since, according to Eq. (4.46),

$$
H^{\prime}(0)-0 \text { and } H^{\prime \prime}(0)-2 \gamma>0,
$$

Property $\mathrm{C}$, in Appendix $\mathrm{C}$, implies that bifurcation is subcritical for both circles and rectangles.

(C) As in Sec. III A, we may try to find a Lyapunov function, but a similar procedure does not seem to give satisfactory results now. Thus we are unable to prove convergence to the set of the steady states.

\section{CONCLUSIONS}

We have considered in Secs. II and III, the combined effects of vertical vibration and gravity in a large aspect ratio container in inverted position, namely, with gravity acting downwards. The linear stability of the flat, rigid body oscillatory state was considered in Sec. II, where we oblained the inslability thresholds for both short-wave and long-wave perturbations. The latter analysis was based on a standard longwave approximation (small horizontal gradients of the variables) that applies in the bulk, outside a boundary layer near the lateral wall, which was analyzed in Appendix A to obtain the appropriate boundary conditions for the solution in the bulk. The resulting $2 \mathrm{D}$ linear eigenvalue problem was (of fourth order and thus) somewhat nonstandard and of independent interest; it was analyzed in Appendix B. The marginal instability curves associated with short- and long-wave perturbations gave a nonvoid stability region in the parameter space (Figs. 2-4) similar to the one already found experimentally in Ref. [9]. In particular, we have shown that the stabilizing effect of vibration is similar to that of the surface tension, and more and more cffective as the forcing frequency increases. Thus the forcing frequency has been assumed to be approprialcly large (namely, the forcing period small as compared to the viscous time) in the remaining part of the paper.

A weakly nonlinear, long wave approximation has been made in Sec. III, where an evolution equation for the free surface in the absence of dry spots was obtained that applies below the upper instability curve in Fig. 2 (and outside a neighborhood of this curve); the latter condition implies that short-wave perturbations are damped out exponentially and can be ignored. This equation admits a Lyapunov function that assures convergence to the set of the steady states. In addition, we analyzed local bifurcation near the instability threshold and showed that this is transcritical for generic containers, and subcritical for some reflection symmetric (such as circular and rectangular) cross sections. The numerical integration of this evolution equation, to obtain further properties of the associated dy namics, is outside the scope of 
this paper; their interest would be considerable if a comparison with experiments were possible. But to our knowledge (and surprisingly to some extend) no further experiments, in addition to those by Wolf $[8,9]$ are available in the literature.

The analysis in Secs. II and III was extended in Sec. IV to the case of a heavy fluid layer over an immiscible lighter one. The instability threshold was obtained under general assumptions, but for simplicity the evolution equation accounting for weakly nonlinear dy namics was made only in two limiting cases, namely, (a) when the viscosity of one of the liquids is negligible and (b) when both viscosities and densities are almost equal. The evolution equation was quite similar (and exhibited similar properties) to that of a single layer in case (a), but it was somewhat different (e.g., it was nonlocal) in case (b).

For illustration we have considered in Sec. II B, a realistic example (an inverted container $5 \mathrm{~cm}$ wide filled with mineral oil) in which the RT instability produced by the earth gravity can be counterbalanced by a $10^{2} \mathrm{~Hz}$ vertical vibration of the container. The required vibrating frequency is much smaller if either the container is smaller or microgravity conditions are considered. Similar applications could be made for the two lluid layers case considered in Scc. IV, but they have been omitted because there are no experiments in the litcrature to compare with. These would be of great interest once the [fairly simple, as compared to the original problem $(1.1)-(1.7)]$ theory in this paper is available.

\section{ACKNOWLEDGMENTS}

This research was partially supported by DGES under Grant No. PB97-0556.

\section{APPENDIX A: THE BOUNDARY LAYERS ATTACHED TO THE SIDE WALLS}

For the sake of brevity and clarity, these boundary layers are analyzed in detail only in the basic limil considered in $\mathrm{Sec}$. IIB. The analysis in the remaining limits is completely similar as will be remarked at the end of this appendix. The structure of this boundary layer is somewhat nonstandard because, as it can be anticipated from Eqs. (2.26) and (2.30). the normal component of the oscillatory velocity at the edge of this boundary layer is non-zero at leading order. This requires that the oscillatory velocity remains of the same order in the boundary layer as in the bulk and, consequently, the oscillatory pressure and free surface deflection are much larger in the boundary layer (where the oscillatory velocity is not almost horizontal, as it is in the bulk) than in the bulk. Thus we seck the cxpansions [cf. Eq. (2.19)]

$$
\begin{gathered}
u=L^{-1} u_{o j} e^{i \omega t}+L^{-2} \text { h.o.h. }+ \text { c.c. }+I^{-4} u_{s i 0}+\cdots, \\
v-L^{-1} v_{o 0} e^{i \omega t}+L^{-2} \text { h.o.h. }+ \text { c.c. }+L^{-\xi} v_{s 0}+\cdots, \\
w=L^{-1} w_{o j} e^{i \omega t} \mid L^{-2} \text { h.o.h. I c.c. }\left|L^{-4} w_{s i}\right| \cdots,
\end{gathered}
$$

$$
\begin{aligned}
& p-\left[p_{o 0}+L{ }^{1} p_{o 1}\right] e^{i m t}+L{ }^{2} \text { h.o.h. }+ \text { c.c. }+L{ }^{1} p_{s 0}+L{ }^{2} p_{s 1} \\
& +I^{-3} p_{s 2}+\cdots, \\
& f-L \quad{ }^{1} f_{o r} e^{i m t}+L{ }^{3} \text { h.o.h. }+ \text { c.c. }+f_{s 0}+L^{1} f_{s 1}+\cdots,
\end{aligned}
$$

where

$$
\eta-s / L
$$

$\xi$ and $s$ are coordinates along the outward unit normal to $\partial \Omega$ and along $\partial \Omega$, respectively, and $u$ and $v$ denote the associated components of the horizontal velocity $\mathbf{u}$. Here $f_{o j}$ and $f_{s j}$ are allowed to depend only on $\xi, \eta$ and $\widetilde{t}$ [defined by Eq. (2.18), as in Scc. II B], and $u_{o j}, u_{s j}, v_{o j}, v_{z j}, w_{o j}, w_{s j}, p_{o j}$ and $p_{s j}$ are allowed to also depend on $z$. Substitution of these cxpansions into Eqs. (2.1)-(2.5) yiclds

$$
\begin{gathered}
p_{o 0 t \xi}-p_{o 01 z}-p_{s 01 \xi}-p_{s(t z}-p_{s i \eta \eta}-p_{s 1 \xi}-p_{s 1 z}-p_{s 1 \eta}-0 \\
u_{o 0 \xi}+w_{o 0 z}-0, u_{o 0 \xi \xi}+u_{o 0 z z}-p_{o 1 \xi}-i \omega u_{o 0} \\
-w_{o 0 \xi \xi}+w_{o 0 z z}-p_{o 1 z}-i \omega w_{o 0}-0
\end{gathered}
$$

if $\infty<\xi<0$ and $0<z<1$, with boundary conditions

$$
\begin{gathered}
u_{o 0}=w_{o 0}=0, \text { if } z=1 \text { or if } \xi=0, \\
w_{o 0}-i \omega f_{o 0}-u_{o t 0 z}+w_{o 0)}-0 \text { if } z-0, \\
p_{o 1}-a \omega^{2} f_{s 1} / 2+C{ }^{2} f_{o 0 \xi \xi}+2 w_{o 0 z}-0 \text { if } z-0, \\
p_{o 01}-a \omega^{2} \int_{s 0} / 2-f_{s 0 \xi \xi} \\
--p_{s 0}+a \omega^{2}\left(f_{o 0}+\bar{f}_{o 0}\right) / 2+C{ }^{2} f_{s 1 \xi \xi} \\
-0 \text { if } z-0, \\
f_{o 0}-0, f_{s 0 \xi}-f_{s 1 \xi}+\widetilde{D}_{0} f_{s 0 \tilde{t}}-0 \circ f_{s 0}-0, \text { if } \xi-0,
\end{gathered}
$$

where

$$
\widetilde{D}_{0}=D / L^{3} .
$$

The problems giving $v_{o 0}$ and $\left(u_{s 0}, v_{s 0}, w_{s 0}\right)$ are decoupled and need not be considered. Using Eqs. (A3), (A8), and (A9) we obtain

$$
p_{o 0}=p_{o 00}(\eta, \tilde{t}), p_{s 0}=p_{s 0}(\tilde{t}), f_{s 0}=f_{s 0}(\eta, \tilde{t}),
$$

where

$$
f_{s 0}-0
$$

if the second boundary condition (2.5) applies; in this case, we only need to apply matching conditions with the solution in the bulk to obtain the Dirichlet boundary condition in Eq. (2.26).

If instead the first boundary condition (2.5) applies then we obtain Eq. (2.27) as follows. The oscillatory velocity components $u_{00}$ and $w_{00}$ are given by Eqs. (A4)-(A7) and 
the following matching conditions with the solution in the bulk [compare Eq. (2.19) with Eq. (Al) and use Eq. (2.30)],

$$
\begin{gathered}
u_{o j}-i(a \omega / 2)\left[1-(\cosh \sqrt{i \omega})^{-1} \cosh \sqrt{i \omega} z\right] f_{s 1 \xi} \rightarrow 0, \\
f_{o f} \rightarrow 0 \text { and } w_{o f} \rightarrow 0 \text { as } \xi \rightarrow-\infty
\end{gathered}
$$

Using this and Eq. (A6) in the equation that results when the continuity equation in Eq. (A4) is integrated in $-\infty<\xi<0$. $0<z<1$, we oblain

$$
\int_{-\infty}^{0} f_{o 0} d \xi+\frac{a}{2}\left(1-\frac{\sinh \sqrt{i \omega}}{\sqrt{i \omega} \cosh \sqrt{i \omega}}\right) \lim _{\xi \rightarrow \infty} f_{s 1 \xi}-0 .
$$

On the other hand, matching conditions with the solution in the bulk require that $f_{s 1 \xi}$ be bounded as $\xi--\infty$, which invoking Eq. (A3) and the last expression in (A8) yields

$$
p_{s 0}-0 \text {. }
$$

Thus we only need to integrate the last equation in Eq. (A8) and use Eq. (A9) to obtain

$$
\lim _{\xi \rightarrow-\infty} f_{s 1 \xi}=\tilde{D}_{02} f_{s 0 \tilde{t}} \mid\left(\alpha C^{2} \omega^{2} / 2\right) \int_{-\infty}^{0}\left(f_{o 0} \mid \overline{f_{o 0}}\right) d \xi,
$$

and invoking Eq. (Al0) we oblain

$$
\lim _{\xi \rightarrow-\infty} f_{s 1 \xi^{-}}-\tilde{D} f_{s 0 \tilde{t}}
$$

where $\widetilde{D}=2 \widetilde{D}_{0} /\left[2 \mid a^{2} \omega^{2} C^{2} \phi(\omega)\right]$, with the function $\phi$ as defined in Eq. (2.28). And we only need to apply matching conditions with the solution in the bulk to obtain the Neulmann boundary condition in Eq. (2.26).

The analysis above stands as $\omega \rightarrow \infty$ and as a weak nonlinearity (as that in Sec. III) is included; thus the boundary conditions (3.9) follows. And the analysis is straightforwardly exlended when a lower liquid layer is added, as in Sec. IV, to obtain the boundary conditions (4.19).

\section{APPENDIX B: LINEAR STABILITY OF THE FLAT STATE}

The stability of the flat state $f_{s}=0$ of Eqs. $(2.35)$ and (2.36) is analyzed as usually, by replacing $f_{s}$ by $F(\tilde{x}, \tilde{y}) e^{\tilde{\mu} \tilde{t}}$ to obtain the linear eigenvalue problem

$$
\begin{gathered}
\tilde{\Delta} U=\mu F, \widetilde{\Delta} F \mid \lambda F=U \text { in } \widetilde{\Omega}, \\
\partial F / \partial \tilde{n}--\mu \beta F \text { or } F-0, \partial U / \partial \tilde{n}-0 \text { on } \partial \widetilde{\Omega}, \\
\int_{\tilde{\Omega}} F d \tilde{x} d \tilde{y}-0,
\end{gathered}
$$

where

$$
\lambda-2 B L^{2} /\left[2+a^{2} \omega^{2} \phi(\omega) C^{2}\right] .
$$

$$
\mu-6 C^{2} L^{4} \tilde{\mu} /\left[2+a^{2} \omega^{2} \phi(\omega) C^{2}\right] . \quad \beta-D /\left(6 C^{2} L^{3}\right) .
$$

If the second equation in Eq. (B1) is substituted into the first one then we obtain a fourth order, linear eigenvalue problem. But instead, for convenience, we consider the linear problem posed by the first equation in Eq. (B 1) and the second boundary condition in Eq. (B2), which uniquely provides $U$ in terms of $F$, in the form

$$
V-\mu G\left(F^{\prime}\right)+\text { const }
$$

where $\mathcal{G}$ is the Green operator associated with the problem

$$
-\widetilde{\Delta} U-F \text { in } \widetilde{\Omega}, \partial U / \partial \tilde{n}-0 \text { on } \partial \widetilde{\Omega}, \int_{\tilde{\Omega}} U d \tilde{x} d \tilde{y}-0,
$$

defined as $\mathcal{G}\left(F^{\prime}\right)-U$. Note that $\mathcal{G}$ is a self-adjoint, compact operator in $X-\left\{F \in L_{2}(\widetilde{\Omega}): \int_{\widetilde{\Omega}} F^{2} d \tilde{x} d \tilde{y}-0\right\}$. Also $\mathcal{G}$ is positive, namely, $\int_{\tilde{\Omega}} F \mathcal{G}(F) d \tilde{x} d \tilde{y} \geqslant 0$. In fact.

$$
\int_{\tilde{\Omega}} F \mathcal{G}(F) d \tilde{x} d \tilde{y}>k_{0} \int_{\tilde{\Omega}}|F|^{2} d \tilde{x} d \tilde{y}
$$

where $k_{0}>0$ is the lowest eigenvalue of $\mathcal{G}$; or, equivalently, $k_{0}{ }^{1}>0$ is the lowest, strictly positive eigenvalue of $\widetilde{\Delta}$ in $\widetilde{\Omega}$, with Neumann boundary conditions at $\partial \widetilde{\Omega}$.

Now, replacing Eq. (B3) into the second equation in Eq. (B1) and using Eq. (B2) we rewrite Eqs. (B1) and (B2) as

$$
\begin{gathered}
\widetilde{\Delta} F+\lambda F-\mu \mathcal{G} F+\text { const in } \widetilde{\Omega} \\
\partial F^{\prime} / \partial \tilde{n}--\mu \beta F^{\prime} \text { or } F-0 \text { on } \partial \widetilde{\Omega} \\
\int_{\tilde{\Omega}} F d \tilde{x} d \tilde{y}-0 .
\end{gathered}
$$

Thus $\mu$ can be also calculated as a generalized eigenvalue of this problem. Since $\mathcal{G}$ is compact, self-adjoint and satisfies Eq. (B5), the spectrum of this problem is readily seen to be real, discrete and bounded above [26]. And using standard variational arguments $[26,27]$, the largest eigenvalue of this problem is found to be given by

$$
\begin{gathered}
-\mu_{0}-\min _{F=Y_{1}} \frac{\int_{\tilde{\Omega}}\left[|\tilde{\nabla} F|^{2} \lambda F^{2}\right] d \tilde{x} d \tilde{y}}{\int_{\tilde{\Omega}} F(F) d \tilde{x} d \tilde{y}+\beta \int_{d \tilde{\Omega}} F^{2} d s} \text { with } \\
Y_{1}-\left\{F \in I^{1}(\tilde{\Omega}): \int_{\tilde{\Omega}} F d \tilde{x} d \tilde{y}-0\right\}
\end{gathered}
$$

if the first boundary condition in Eq. (B6) holds, where $s$ is an arch length parameter along $\partial \widetilde{\Omega}$ and $H^{\prime}(\widetilde{\Omega})$ is the Sobolev space of those functions that, together with their first partial derivatives, are square integrable in $\widetilde{\Omega}$. And 


$$
\begin{gathered}
-\mu_{0}-\min _{F=Y_{2}} \frac{\int_{\tilde{\Omega}}\left[|\tilde{\nabla} F|^{2} \lambda F^{2}\right] d \tilde{x} d \tilde{y}}{\int_{\tilde{\Omega}} F \mathcal{G}\left(F^{2}\right) d \tilde{x} d \tilde{y}} \text { with } \\
Y_{2}=\left\{F \in H^{\prime}(\tilde{\Omega}): \int_{\tilde{\Omega}} F d \tilde{x} d \tilde{y}=0, F=0 \text { on } \partial \tilde{\Omega}\right\}
\end{gathered}
$$

if the second boundary condition in Eq. (B6) holds. Note that because $\beta \geqslant 0$ and $\mathrm{Eq}$. (B5) holds, the functionals that are minimized in Eqs. (B7) and (B8) are bounded and continuous (in fact, analytic). Since, in addition (a) $\mu-0$ is an eigenvalue of Eq. (B6) if and only if

$\widetilde{\Delta} F \mid \lambda F=$ const in $\widetilde{\Omega}, \partial F / \partial \tilde{n}=0$ or $F=0$ on $\partial \widetilde{\Omega}$,

$$
\int_{\tilde{\Omega}} F d \tilde{x} d \tilde{y}=0
$$

has a nontrivial solution, and (b) the lowest eigenvalue of this problem is given by

$$
\lambda_{0}-\min _{f i r \gamma_{1}} \frac{\int_{\tilde{\Omega}}|\tilde{\nabla} F|^{2} d \tilde{x} d \tilde{y}}{\int_{\tilde{\Omega}} F^{2} d \tilde{x} d \tilde{y}} \text { or } \lambda_{i j}-\min _{\mu r<\gamma_{2}} \frac{\int_{\tilde{\Omega}}|\tilde{\nabla} F|^{2} d \tilde{x} d \tilde{y}}{\int_{\tilde{\Omega}} F^{2} d \tilde{x} d \tilde{y}},
$$

depending on whether Eq. (B7) or (B8) applies, we obtain the following property, which is the object of this appendix.

Property B1. If $\lambda<\lambda_{0}$ then all eigenvalues of Eqs. (B1) and (B2) are strictly negative, and if $\lambda>\lambda_{0}$ then Eqs. (B1) and (B2) possesses a strictly positive eigenvalue.

Proof. The first assertion follows from Eqs. (B5), (B7) and (B8), and (B10). And the second assertion follows from the first one because, according to the characterization (B7)(B8), $\mu_{0}$ (i) depends continuously on $\lambda$ and (ii) strictly increases as $\lambda$ increases.

\section{APPENDIX C: LOCAL BIFURCATION FROM THE FLAT STATE AT THE INSTABILITY THRESHOLD}

Here we consider the general problem

$$
\begin{aligned}
& {[1-H(f)] \widetilde{\Delta} f+\lambda f-H^{\prime}(f)|\widetilde{\nabla} f|^{2} / 2-\text { const, in } \widetilde{\Omega},} \\
& \partial f / \partial \tilde{n}-0 \text { or } f-0 \text { on } \partial \widetilde{\Omega}, \int_{\widetilde{\Omega}} f d \tilde{x} d \tilde{y}-0,
\end{aligned}
$$

where $/ /$ is a $C^{\infty}$ function such that

$$
H(0)=0 .
$$

For appropriate // this problem includes as particular cases those providing the stcady states of Eqs. (3.14) and (3.15), (4.29) and (4.30), and (4.42) and (4.43). The lincarization of Eqs. (C1) and (C2) around the solution $f=0$ leads to Eq.
(B9). Let us consider a simple eigenvalue of this linear problem, $\lambda_{0}$. Local bifurcation of Eqs. (3.3) and (3.4) at $\lambda=\lambda_{0}$ is readily analyzed by the Lyapunov-Schmidt method [28] as follows. Let us replace Eqs. $(\mathrm{C} 1)$ and $(\mathrm{C} 2)$ by

$$
\begin{aligned}
& {[1-H(f)] \widetilde{\Delta} f+\lambda f-I^{\prime}(f) \mid \widetilde{\nabla} f^{2} / 2-\text { const }+\Gamma(\varepsilon, \varsigma) F_{0},} \\
& \text { with } f^{-} \varsigma\left(f_{0}+\psi /\right) \text { and } \lambda-\lambda_{0}+\varepsilon \text {, in } \widetilde{\Omega} \text {, } \\
& \partial f / \partial \tilde{n}=0 \text { or } f=0 \text { on } \partial \tilde{\Omega} \text {, } \\
& \int_{\tilde{\Omega}} \int d \tilde{x} d \tilde{y}-\int_{\tilde{\Omega}} \psi F_{0} d \tilde{x} d \tilde{y}-0
\end{aligned}
$$

where $F_{i}$ is an eigenfunction of (B9) associated with the eigenvalue $\lambda_{0}$, such that

$$
\int_{\tilde{\Omega}} F_{t}^{2} d \tilde{x} d \tilde{y}=1
$$

This problem coincides with Eqs. (C1) and (C2) if

$$
\Gamma(\varepsilon, \varsigma)-0
$$

thus this equation is called bifurcation equation. Now, the extension of the implicit function theorem to Banach spaces [29], applied in an appropriate function space [e.g., the space $H_{B}^{2}(\widetilde{\Omega})$ of those functions that together with their first and second spatial derivatives are in $L_{2}(\widetilde{\Omega})$ and satisfy Eq. (C5)]. implies that Eqs. (C4) and (C5) uniquely provides $\psi$ and $\mathrm{I}^{\text {', }}$ as $C^{s}$ functions of $\varepsilon$ and $s$, for all sufficiently small $\varepsilon$ and $s$, and that $\psi-0$ and $\Gamma-0$ if $s-0$. Then those functions can be written, through a Taylor expansion, as

$$
\begin{aligned}
& \psi=s\left(\varepsilon \psi_{1}\left|s \psi_{2}\right| s^{2} \psi_{3} \mid \cdots\right), \\
& \Gamma=s\left(\varepsilon \Gamma_{1}\left|s \Gamma_{2}\right| s^{2} \Gamma_{3} \mid \cdots\right),
\end{aligned}
$$

and substitution of these into Eqs. (C4) and (C5) yields

$$
\widetilde{\Delta} \psi_{1}+\lambda_{0} \psi_{1}-\left(\mathrm{I}_{1}-1\right) F_{0}+\text { const. }
$$

$$
\widetilde{\Delta} \psi_{2}+\lambda_{0} \psi_{2}-\mathbf{I}_{2} F_{0}+H^{\prime}(0)\left(\left|\widetilde{\nabla} F_{0}\right|^{2} / 2+F_{0} \widetilde{\Delta} F_{0}\right)+\text { const }
$$

$\widetilde{\Delta} \psi_{3}\left|\lambda_{0} \psi_{3}=\Gamma_{3} F_{0}\right| H^{\prime}(0)\left(\widetilde{\nabla} \psi_{2}, \widetilde{\nabla} F_{0}\left|F_{0} \widetilde{\Delta} \psi_{2}\right| \psi_{2} \widetilde{\Delta} F_{0}\right)$

$$
\begin{aligned}
& +H^{\prime \prime}(0)\left(F_{0}^{2} \widetilde{\Delta} F_{0}+F_{00}\left|\widetilde{\nabla} F_{00}\right|^{2}\right) / 2 \\
& + \text { const in } \widetilde{\Omega},
\end{aligned}
$$

$$
\begin{gathered}
\partial \psi_{1} / \partial \tilde{n}-\partial \psi_{2} / \partial \tilde{n}-\partial \psi_{3} / \partial \tilde{n}-0 \text { or } \\
\psi_{1}-\psi_{2}-\psi_{3}-0 \text { on } \partial \widetilde{\Omega}
\end{gathered}
$$



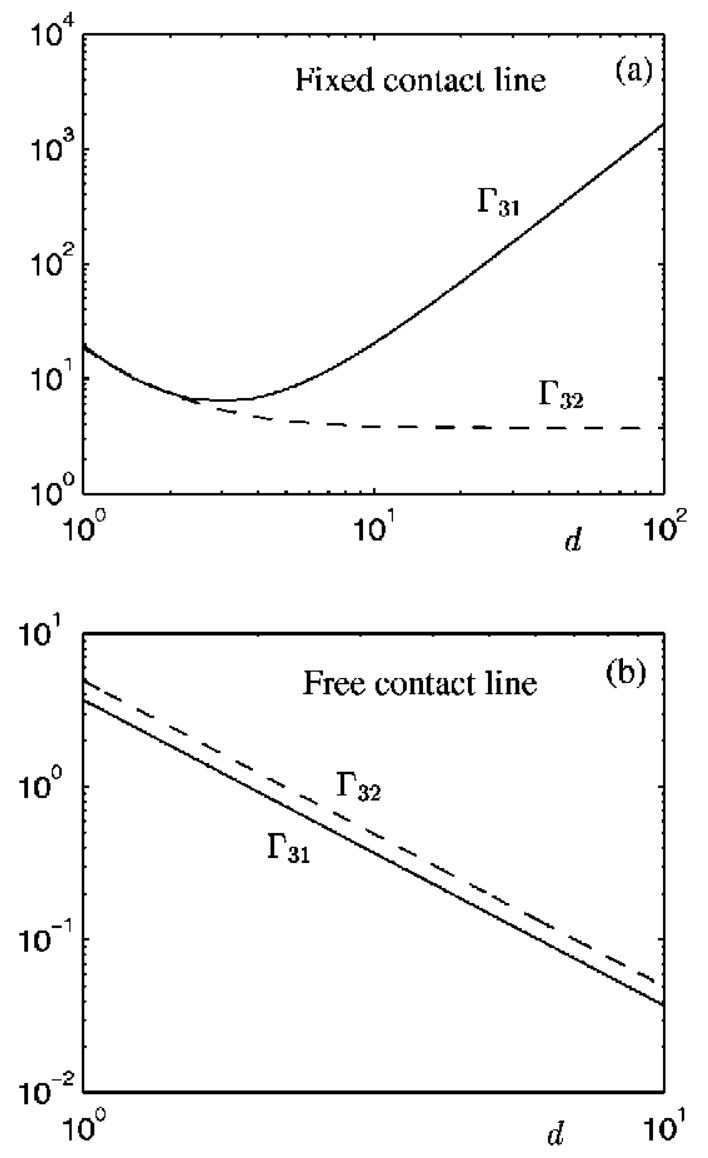

liGG. 5. The constants appearing in Iiq. (C18) when $\widetilde{\Omega}$ is the square of sides 1 and $d$. The atlachment mode of the contact line (free or lixed) is indicaled.

$$
\begin{aligned}
\int_{\tilde{\Omega}} \psi_{1} d \tilde{x} d \tilde{y} & =\int_{\tilde{\Omega}} F_{0} \psi_{1} d \tilde{x} d \tilde{y}=\int_{\tilde{\Omega}} \psi_{2} d \tilde{x} d \tilde{y} \\
& =\int_{\tilde{\Omega}} \psi_{2} F_{0} d \tilde{x} d \tilde{y}=\int_{\tilde{\Omega}} \psi_{\tilde{3}} d \tilde{x} d \tilde{y} \\
& =\int_{\tilde{\Omega}} \psi_{3} F_{0} d \tilde{x} d \tilde{y}=0 .
\end{aligned}
$$

These three nonhomogeneous, singular linear problems are readily secn to be associated with self-adjoint operators, and they have a solution if and only if the right hand sides of the three equations in Eqs. (C9)-(C11) are orthogonal to $F_{0}$, with the inner product of $L_{2}(\widetilde{\Omega})$. This solvability condition yields

$$
\begin{gathered}
\Gamma_{1}-1, \\
\Gamma_{2}--H^{\prime}(0) \int_{\tilde{\Omega}} F_{0}\left(\left|\tilde{\mathbf{v}} F_{0}\right|^{2} / 2+F_{0} \widetilde{\Delta} F_{0}\right) d \tilde{x} d \tilde{y} \\
-3 H^{\prime}(0) \lambda_{0} \int_{\tilde{\Omega}} F_{0}^{3} d \tilde{x} d \tilde{y} / 4,
\end{gathered}
$$

$$
\begin{aligned}
\Gamma_{3}= & \int_{\tilde{\Omega}} F_{0}\left[H^{\prime}(0)\left(\widetilde{\boldsymbol{\nabla}} \psi_{2} \cdot \widetilde{\boldsymbol{\nabla}} F_{0}\left|F_{0} \widetilde{\Delta} \psi_{2}\right| \psi_{2} \widetilde{\Delta} F_{0}\right)\right. \\
& \left.+H^{\prime \prime}(0)\left(F_{0}^{2} \widetilde{\Delta} F_{0}+F_{0}\left|\widetilde{\nabla} F_{0}\right|^{2}\right) / 2\right] d \tilde{x} d \tilde{y},
\end{aligned}
$$

where we have taken into account Eq. (B9) and the expression

$$
\begin{aligned}
\lambda_{0} \int_{\tilde{\Omega}} F_{0}^{3} d \tilde{x} d \tilde{y} & =\int_{\tilde{\Omega}} F_{0}^{2} \tilde{\Delta} F_{0} d \tilde{x} d \tilde{y} \\
& =\int_{\tilde{\Omega}}\left(\tilde{\nabla} F_{0}\right) \cdot\left[\tilde{\nabla}\left(F_{0}^{2}\right)\right] d \tilde{x} d \tilde{y} \\
& -2 \int_{\tilde{\Omega}} F_{0}\left|\tilde{\nabla} F_{0}\right|^{2} d \tilde{x} d \tilde{y} .
\end{aligned}
$$

which follows from Eq. (B9) upon integration by parts. Thus $\Gamma_{2}$ is generically (for domains of arbitrary shape) nonzero and, according to $\mathrm{Eq}$. (C8), the bifurcation is generically transcritical. But for some symmetric domains, like the circles and rectangles considered in Sec. II, if $\lambda_{i}$ is the lowest eigenvalue of Eq. (B9), then $F_{0}$ is antisymmetric and $\Gamma_{2}$ vanishes. In this case, $\psi_{2}$ is uniquely given by

$$
\psi_{2}=H^{\prime}(0) \Psi
$$

where $\Psi$ is the unique solution of

$$
\begin{gathered}
\tilde{\Delta} \Psi+\lambda_{0} \Psi-\left|\widetilde{\nabla} F_{0}\right|^{2} / 2+F_{0} \widetilde{\Delta} F_{0}+\text { const in } \widetilde{\Omega}, \\
\partial \Psi / \partial \tilde{n}=0 \text { or } \Psi=0 \text { on } \partial \tilde{\Omega}, \\
\int_{\tilde{\Omega}} \Psi d \tilde{x} d \tilde{y}-\int_{\tilde{\Omega}} \Psi F_{0} d \tilde{x} d \tilde{y}-0 .
\end{gathered}
$$

And invoking Eq. (C13) we obtain

$$
\Gamma_{3}=\left[H^{\prime}(0)\right]^{2} \Gamma_{31} \mid H^{\prime \prime}(0) \Gamma_{32} / 2,
$$

where the constants $\Gamma_{\$ 1}$ and $\Gamma_{32}$ are given by

$$
\begin{gathered}
\Gamma_{31}-\int_{\tilde{\Omega}} F_{0}\left(\tilde{\nabla} \Psi \cdot \widetilde{\nabla} F_{0}+F_{0} \widetilde{\Delta} \Psi+\Psi \widetilde{\Delta} F_{0}\right) d \tilde{x} d \tilde{y} \\
\mathbf{I}_{32}-\int_{\tilde{\Omega}} F_{0}\left(F_{0}^{2} \widetilde{\Delta} F_{0}+F_{0}\left|\widetilde{\nabla} F_{0}\right|^{2}\right) d \tilde{x} d \tilde{y} / 2
\end{gathered}
$$

and depend only on the domain $\widetilde{\Omega}$ and on the boundary attachment mode of the contact line, namcly, on which boundary condition is used in Eq. (3.7). In particular, if $\widetilde{\Omega}$ $=\widetilde{\Omega}_{1}$ is the circle of diameter 1 , then

$$
\Gamma_{31} \simeq 11.42 \text { and } \Gamma_{32} \simeq 14.78
$$

for Prec contact line, and

$$
\Gamma_{31} \simeq 4.20 \text { and } \Gamma_{32} \simeq 3.79
$$

for fixed contact line, as obtained from Eq. (C13), where $F_{0}$ is to be taken from Eqs. (2.16) and (2.17) [and rescaled to 
satisfy Eq. (C6)] and $\psi_{2}$ is obtained numerically from Eqs. (C14)-(C16). Similarly, if $\widetilde{\Omega}-\widetilde{\Omega}_{2}$ is the square of sides 1 and $d \geqslant 1$ then these two constants are found to be as plotted $v$ s $d$ in Fig. 5.

Now, according to Eqs. (C19) and (C20) and Fig. 5, the constants $\Gamma_{31}$ and $\Gamma_{32}$ are strictly positive in both circles and rectangles, for both free and fixed contact lines. And using (C17), $\Gamma_{3}>0$ in all these cases if $/ I^{\prime \prime}(0) \geqslant 0$. And, according to $(\mathrm{C} 8)$ and the first expression in $(\mathrm{C} 12)$, if $\mathrm{I}_{3}>0$ then the bifurcated solutions [given by the bifurcation equation (C7)] exist for $\varepsilon \equiv \lambda \quad \lambda_{0}<0$, which means (Property B1) that the bifurcation is subcritical. Thus we have the following

Property C1. If $H^{\prime \prime}(0) \geqslant 0$ and $\widetilde{\Omega}$ is either a circle or $a$ rectangle, then the bifurcation at $\lambda-\lambda_{0}$ is a subcritical pitchfork one.
[1] J.W.S. I ord Rayleigh, in Scientific Papers (Cambridge University Press, Cambridge, England, 1900), Vol. II, pp. 200 207.

$\lceil 2\rceil$ G.I. Taylor, Proc. R. Soc. London, Ser. $\Lambda$ 201, 192 (1950).

[3] S. Chandrasckhar, Hydrodynamic and Hydromagnetic Stability (Oxford Univ. Press, London, 1961).

[4] H.J. Kull, Phys. Rep. 206, 197 (1991).

[5] J.D. Buckmaster, Physica D 12, 173 (1984).

[6] J.D. Lindl, Inertial Confinement Fusion (Springer-Verlag, New York, 1998).

[7] B.A. Remington, R.P. Drake, H. Takabe, and D. Amet, Phys. Plasmas 7, 1641 (2000).

[8] (ì.Il. Wolf, \%. Phys. 227, 291 (1969).

[9] (ì.I]. Wolf, Phys. Rev. I,ett. 24, 444 (1970).

[10 $\mathrm{l}$. Troyon and R. Gruber, Phys. lluids 14, 2069 (1971).

[11] I.M. J Ioffmann and (3.ll. Wolf. J. Appl. Phys. 45, 3859 (1974)

$\lceil 12\rceil$ G.W. Young and S.H. Davis, J. Fluid Mcch. 174, 327 (1987).

「13ך D.M. Henderson and J.W. Miles, J. Fluid Mech. 275, 285 (1994).

$\lceil 14\rceil$ M. Faraday, Philos. Trans. R. Soc. London 121, 319 (1831).

「15† J. Miles and D. Henderson, Annu. Rev. Fluid Mech. 22, 143 (1990)

[16] M.C. Cross and P.C. Hohenberg, Rev. Mod. Phys. 65, 851 (1993)

[17] S. Fauve, in Dynamics of Nonlinear and Disordered Systems, edited by G. Martinez. Mekler and T.II. Seligman (World Scientific, Singapore, 1995), pp. 67-115.

[18] K. Kumar and L.S. Tuckerman, J. Fluid Mech. 279, 49 (1994).

「19] O.A. Ladyženskaja, V.A. Solonnikov, and N.N. Ural'ceva, Linear and Quasilinear Liquations of l'arabolic Type (American Mathematical Society. Providence, 1968).

[20] D, Henry. Geometric Theory of Semilinear Parabolic Equations (Springer-Verlag. New York, 1989).

$\lceil 21\rceil$ R. Temam, Infunite-Dimensional Systems in Mechanics and Physics (Springer-Verlag. New York, 1988).

[22] J.K. Hale, Asymptotic Behavior of Dissipative Systems (American Mathematical Socicty, Providence, 1989).

$\lceil 23\rceil$ R.A. Adams, Soboley Spaces (Academic Press, New York, 1975).

[24] O.A. Ladyženskaja and N.N. Ural'ceva, Linear and Quasilinear Elliptic Equations (Academic Press, New York, 1968).

「25] D. Gilbarg and N.H. Trudinger, Elliptic Partial Differential liqualions of Second Order (Springer-Verlag. New York, 1985).

「26ך E. Kreyszig, Introductory Functional Analysis with applications (John Wilcy, New York, 1978).

[27] R. Courant and I). I libert, Methods of Mathematical Mhysics, Vols. I and II. (Interscience, New York, 1962).

[28] S.N. Chow and J.K. Hale, Methods of Bifurcation Theory (Springer-Verlag, New York, 1982).

[29] J. Dieudonne, Foundations of Modern Analysis (Academic Press, New York, 1960). 